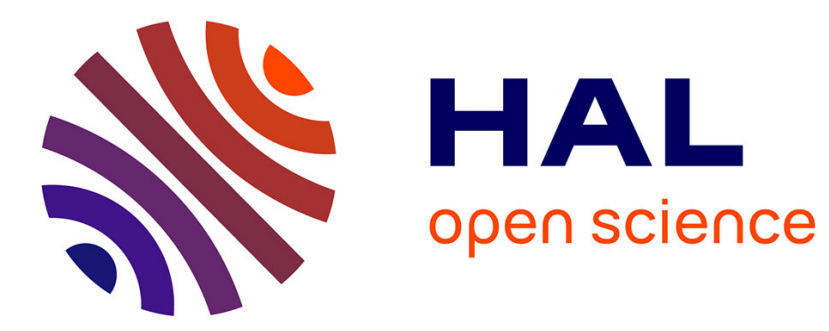

\title{
ANALYSIS OF THE INCOMPATIBILITY OPERATOR AND APPLICATION IN INTRINSIC ELASTICITY WITH DISLOCATIONS
}

Samuel Amstutz, Nicolas van Goethem

\section{- To cite this version:}

Samuel Amstutz, Nicolas van Goethem. ANALYSIS OF THE INCOMPATIBILITY OPERATOR AND APPLICATION IN INTRINSIC ELASTICITY WITH DISLOCATIONS. SIAM Journal on Mathematical Analysis, 2016, 48 (1), pp.320-348. hal-01150071v2

\section{HAL Id: hal-01150071 \\ https://hal.science/hal-01150071v2}

Submitted on 4 Nov 2015

HAL is a multi-disciplinary open access archive for the deposit and dissemination of scientific research documents, whether they are published or not. The documents may come from teaching and research institutions in France or abroad, or from public or private research centers.
L'archive ouverte pluridisciplinaire HAL, est destinée au dépôt et à la diffusion de documents scientifiques de niveau recherche, publiés ou non, émanant des établissements d'enseignement et de recherche français ou étrangers, des laboratoires publics ou privés. 


\title{
ANALYSIS OF THE INCOMPATIBILITY OPERATOR AND APPLICATION IN INTRINSIC ELASTICITY WITH DISLOCATIONS
}

\author{
SAMUEL AMSTUTZ AND NICOLAS VAN GOETHEM
}

\begin{abstract}
The incompatibility operator arises in the modeling of elastic materials with dislocations and in the intrinsic approach to elasticity, where it is related to the Riemannian curvature of the elastic metric. It consists of applying successively the curl to the rows and the columns of a 2nd-rank tensor, usually chosen symmetric and divergence free. This paper presents a systematic analysis of boundary value problems associated with the incompatibility operator. It provides answers to such questions as existence and uniqueness of solutions, boundary traces lifting and transmission conditions. Physical interpretations in dislocation models are also discussed, but the application range of these results exceed by far any specific physical model.
\end{abstract}

\section{INTRODUCTION}

The incompatibility operator is a 2nd-order differential operator consisting of taking the curl of the rows and the columns of a 2nd-rank tensor $\epsilon$, viz.,

$$
\text { inc } \epsilon=\operatorname{Curl} \mathrm{Curl}^{\mathrm{t}} \epsilon \text {, }
$$

the curl being taken row-wise. The incompatibility operator arises in physics, in the area of dislocation modeling, since the linear elastic strain $\epsilon$ is incompatible in the presence of dislocations, that is, cannot be written as a symmetric gradient, as soon as inc $\epsilon \neq 0$. Specifically, its incompatibility is related to the tensor-valued density of dislocations $\Lambda$ as found by Kröner [14] and further discussed in, e.g. [20, 23], and shows ultimately as a macroscopic manifestation of plasticity (let us recall that plasticity is generated by dislocation motion). The insight of Kröner was to understand the incompatibility as a genuine geometric property of the dislocated crystal related to the connection torsion and contortion (we refer to $[9,18,20]$ ), the crystallographic evidence of the latter had been first identified by Nye [17]. In a recent contribution [21] to this discussion, it was shown that the incompatible strain writes by virtue of the Beltrami decomposition [15] as

$$
\epsilon=\nabla^{S} u+\operatorname{inc} F,
$$

where $u$ may be given the meaning of a displacement field, here complemented with a tensorvalued symmetric and divergence-free field $F$ which is related to the dislocation density by the formula

$$
\text { inc inc } F=\operatorname{inc} \epsilon=\operatorname{Curl} \kappa \text {, }
$$

where the last equality is due to Kröner [14], and with the contortion tensor

$$
\kappa:=\Lambda-\frac{\mathbb{I}}{2} \operatorname{tr} \Lambda \text {. }
$$

Here $\Lambda$ is the macroscopic counterpart of the mesoscopic dislocation density tensor $\Lambda_{\mathcal{L}}:=$ $\tau \otimes b \mathcal{H}_{\llcorner\mathcal{L}}^{1}$, where $\tau$ is the unit tangent vector to the dislocation line $\mathcal{L}$, and $b$ its Burgers vector.

Moreover, Eq. (1.1) shows tensor $\mathrm{Curl}^{\mathrm{t}} \epsilon$, called the Frank tensor [23] and from which the infinitesimal rotations and the displacement field are classically defined in linear elasticity. In fact, in the presence of dislocation lines, the displacement and rotation jumps around the lines are explicitly given [15] by means of recursive line integration of linear combinations of the elastic strain and Frank tensors. Obviously, these jumps vanish if and only if the strain incompatibility vanishes.

2010 Mathematics Subject Classification. 35J48,35J58,53A05,74A45,74B99.

Key words and phrases. incompatibility, dislocations, intrinsic elasticity, Sobolev spaces, lifting, transmission conditions. 
So far, we can say that incompatibility is an important operator in Continuum mechanics, which is related to dislocations and which carries a clear geometric interpretation. About this latter point, it should be emphasized that inc $\epsilon$ is another way of writing the curvature tensor associated to the elastic metric $g:=\mathbb{I}-2 \epsilon$, to the first order (the explicit relation between the 4th-rank curvature tensor and the 2nd-rank strain incompatibility can be found in [15]), whose properties in mathematical models of elasticity have been discussed in a series of recent works by $\mathrm{Ph}$. Ciarlet (see for instance $[6,7]$ ), in what he calls the intrinsic model. Let us here emphasize the deep impact of this point of view for modeling, since it consists in a change of paradigm: no more to consider the displacement as the main model variable, rather the strain, and from its knowledge (be it given, or be it deduced from a constitutive law from the stress field) to define an elastic metric $g$, the associated Riemann curvature tensor (and possibly, the Cartan torsion, as far as dislocations are considered, see, e.g. [14, 20]), and then in a second step to deduce a displacement field. In Ciarlet's series of works, the displacement is found as soon as the curvature tensor associated to $g$ vanishes. We also share this point of view, and consider the elastic strain as the primal variable, here defined from the stress $\sigma$ by the linear homogeneous, isotropic and isothermal constitutive law

$$
\epsilon=A^{-1} \sigma
$$

where $A$ is the elasticity tensor. However, for us, not only the Riemann curvature does not vanish because of (1.3), but it is the central model variable besides the elastic strain.

The main motivation and purpose of this work is indeed the mathematical study of the incompatibility operator, in particular in terms of function spaces used and their properties. From a mathematical viewpoint, it should also be stressed that the incompatibility operator is related to the Laplacian in the sense that for symmetric and divergence-free fields $E$, one has $\operatorname{tr}$ inc $E=\Delta \operatorname{tr} E^{1}$ and inc inc $E=\Delta^{2}$. Thus, in some sense, it constitutes a tensor generalization of the Laplacian, but as for the Curl Curl operator (recall that Curl Curl $=-\Delta$ for solenoidal vectors), any associated boundary value problem must be addressed carefully since it applies to solenoidal fields and hence might not be an elliptic operator (see [13] for the analysis of Curl Curl systems, the vector and 1st-order counterpart of our study). Indeed, the solution must satisfy the divergence-free condition in the domain, as well as specific boundary conditions (i.e., complementary in the sense of Agmon, Douglis and Nirenberg [1]) normal and/or tangential components of its boundary traces. Note that in $H^{1}$-spaces, the study of divergence-free fields is of the utmost importance in fluid dynamics [10, 19]. In particular, boundary lifiting results can be found in [11].

A first natural question is to seek the appropriate boundary conditions (if one thinks of the strong form) in order to well define the BVP inc (Minc $E)=\mathbb{G}$, or the appropriate function space (if one thinks of the weak form) to have existence of minimizers for $\int_{\Omega}\left(\frac{1}{2} \mathbb{M}\right.$ inc $E$. inc $E-$ $\mathbb{G} \cdot E) d x$, where $\mathbb{G}$ is some given symmetric and divergence-free force dual to $E$. A first issue is therefore the bilinear form coercivity and the function trace lifting properties, that is, given appropriate combinations of $E$ and its normal derivatives, is it possible to find a divergence-free field $E$ in $H^{2}$ whose trace on the boundary corresponds to these values?

These issues are positively answered in this paper. As a first step, a study of the extension of boundary tangent and normal vectors will also be achieved. Such extensions and their differentiability properties can here be found in Theorem 2.2. We emphasize that our solution method is not very standard, since it is coordinate-free and based on the extension of an orthonormal basis on the boundary, which is thus viewed as locally Euclidean, though with a triad of local, non constant, basis vectors. Our core result is Theorem 3.10 and states that one can find $F \in H_{0}^{2}(\Omega)$ with prescribed divergence in $\Omega$. Its main application for our purposes is about trace boundary lifting for solenoidal fields in $H^{2}(\Omega)$. The exact statement can be found in Theorem 3.12. Then, in Section 4, combining this latter result with the bilinear form coercivity in $H_{0}^{2}(\Omega)$ (namely, Theorem 3.9), existence and uniqueness of the nonhomogeneous boundary value problem inc $(\mathbb{M}$ inc $E)=\mathbb{G}$ follow in a standard way. Lastly, with a view to perform

\footnotetext{
$1_{\text {trace }} \operatorname{tr}$ here meaning the sum of the diagonal components of a 2nd-rank tensor.
} 
topological sensitivity analysis in future work in the spirit of $[2,3]$, transmission conditions are identified by means of an appropriate Gauss-Green formula. They are given in Theorem 4.4.

To the knowledge of the authors, our most substantial auxiliary results, namely Theorems 3.10 and 3.12 were not found elsewhere in the literature, and seem of the utmost importance for a broad range of applications, exceeding by far our study of the incompatibility operator. Let us emphasize that the incompatibility operator and its physical interpretation in dislocation modeling must be considered here as one possible application, which was the motivation for addressing this problem, but Theorems 3.10 and 3.12 show a level of generality which we believe renders their study useful to a large community of mathematicians working in applied sciences. Let us also stress the importance of boundary lifting in numerical analysis, in particular in the finite elements methods $[5,11]$.

To conclude, the proper boundary value problem is discussed in Section 4, while its application in dislocation models is proposed in Section 5.

Physical meaning of the model field $E$ in Elasticity. The first physical interpretation of the variable $E$ is the field $F$ in Beltrami decomposition (1.2). Let be given the stress $\sigma$ and define the elastic strain as $\epsilon:=A^{-1} \sigma$, where $A$ is the assumed constant elasticity tensor, i.e., $A=2 \mu \mathbb{I}_{4}+\lambda \mathbb{I}_{2} \otimes \mathbb{I}_{2}$, with $\lambda$ and $\mu$ the Lamé coefficicents. Equilibrium reads

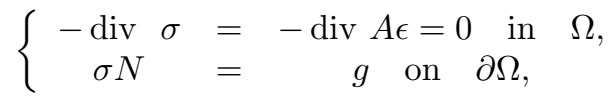

and from (1.2) is rewritten as

$$
\left\{\begin{array}{ccc}
-\operatorname{div}\left(A \nabla^{S} u\right) & =\mathcal{F}_{\Lambda_{\mathcal{L}}}:=\lambda \nabla \operatorname{tr}(\text { inc } F) \text { in } \Omega, \\
\left(A \nabla^{S} u\right) N & =g-\lambda \operatorname{tr}(\operatorname{inc} F) N=g \text { on } \partial \Omega,
\end{array}\right.
$$

recalling the solenoideal property of inc $F$. Therefore, $u$ is called the generalized displacement field. By virtue of (1.3) with a suitable choice of boundary conditions, one also has

$$
\left\{\begin{array}{ccc}
\operatorname{inc} \text { inc } F=\text { inc } \epsilon & = & \text { Curl } \kappa \text { in } \Omega, \\
F & = & 0 \text { on } \partial \Omega \\
\left(\partial_{N} F \times N\right)^{t} \times N & =0 & \text { on } \partial \Omega
\end{array}\right.
$$

Systems (1.6) and (1.7) are also discussed in [21].

Moreover, recall (1.2) and take $E=\epsilon^{0}:=$ inc $F$. By (1.3), the symmetric and solenoidal field $\epsilon^{0}$ is the part of the elastic strain which plays a role with dislocations. Thus it may be called the dislocation-induced elastic strain. Let us address now the question of a thermodynamical setting in our elastic body with dislocations in which the free energy would depend on internal defect variables such as $\epsilon^{0}$ and $\kappa$. Considering a high-order model in the spirit of [4], which involves the derivatives of $\kappa$ in the form of its curl, then by (1.3) the free energy is a function of $\epsilon^{0}$ and inc $\epsilon^{0}$. If now a quadratic model is proposed (we refer again to [4]) one would naturally consider terms such as $\frac{1}{2} \mathbb{M}$ inc $\epsilon^{0}$. inc $\epsilon^{0}$ in the free energy, with $\mathbb{M}$ a positive-definite 4th-rank tensor whose components are related to material properties of the crystal and of the dislocations. Therefore, one is lead to study variational problems of form

$$
\inf _{\epsilon=\nabla^{S} u+\epsilon^{0}} \int_{\Omega}\left(\frac{1}{2} A \nabla^{S} u \cdot \nabla^{S} u+\frac{1}{2} \mathbb{M} \text { inc } \epsilon^{0} \cdot \text { inc } \epsilon^{0}\right) d x-\int_{\Omega} \mathbb{G} \cdot \epsilon d x,
$$

where $\mathbb{G}$ is a body force that works against the total strain $\epsilon$. Of course, surface forces could also be incorporated, as discussed in Section 5. If now $\mathbb{G}$ is decomposed as $\mathbb{G}=\nabla^{S} w+\mathbb{G}^{0}$, ones has

$$
\int_{\Omega} \mathbb{G} \cdot \epsilon d x=\int_{\Omega}\left(-\operatorname{div} \nabla^{S} w \cdot u+\mathbb{G}^{0} \cdot \epsilon^{0}\right) d x
$$

where all boundary terms have again been dropped for simplicity. Thus, $f:=-\operatorname{div} \nabla^{S} w$ is recognized as a body force that works against the displacement, while $\mathbb{G}^{0}$ works against the solenoidal part of the strain. Moreover, the minimizations with respect to $u$ and $\epsilon^{0}$ become uncoupled. The former one provides the standard linear elasticity equations, and the latter one formally yields

$$
\operatorname{inc}\left(\mathbb{M} \text { inc } \epsilon^{0}\right)=\mathbb{G}^{0} \text {. }
$$


With these two examples of physical fields $E$, whose incompatibility plays a central role, let us now begin the mathematical analysis.

Notations and conventions. Let $\Omega$ be a bounded domain of $\mathbb{R}^{3}$ with smooth boundary $\partial \Omega$. By smooth we mean $C^{\infty}$, but this assumption could be considerably weakened. Let $\mathbb{M}^{3}$ denote the space of square 3-matrices, and $\mathbb{S}^{3}$ of symmetric 3-matrices. Divergence, curl, incompatibility and cross product with 2 nd order tensors are defined componentwise as follows with the summation convention on repeated indices. Here, $E$ and $T$ are 2 nd rank tensors, $N$ is a vector, and $\epsilon$ is the Levi-Civita 3rd rank tensor. One has:

$$
\begin{aligned}
(\operatorname{div} E)_{i} & :=\partial_{j} E_{i j}, \\
(\operatorname{Curl} T)_{i j} & :=(\nabla \times T)_{i j}=\epsilon_{j k m} \partial_{k} T_{i m}, \\
(\operatorname{inc} E)_{i j} & :=\left(\operatorname{Curl~Curl~}^{\mathrm{t}} E\right)_{i j}=\epsilon_{i k m} \epsilon_{j l n} \partial_{k} \partial_{l} E_{m n}, \\
(N \times T)_{i j} & :=-(T \times N)_{i j}=\epsilon_{j k m} N_{k} T_{i m}, \\
(E \times T)_{i j k} & :=\epsilon_{j m n} E_{i m} T_{k n}, \\
\operatorname{tr}(E \times T)_{j} & :=\epsilon_{j m n} E_{p m} T_{p n} .
\end{aligned}
$$

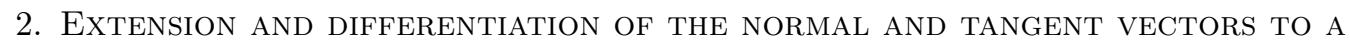
SURFACE

2.1. Signed distance function and extended unit normal. We denote by $N_{\partial \Omega}$ the outward unit normal to $\partial \Omega$, and by $b$ the signed distance to $\partial \Omega$, i.e.,

$$
b(x)=\left\{\begin{aligned}
\operatorname{dist}(x, \partial \Omega) & \text { if } x \notin \Omega \\
-\operatorname{dist}(x, \partial \Omega) & \text { if } x \in \Omega .
\end{aligned}\right.
$$

We recall the following results ([8], Chap. 5, Thms 3.1 and 4.3).

Theorem 2.1. There exists an open neighborhood $W$ of $\partial \Omega$ such that

(1) $b$ is smooth in $W$;

(2) every $x \in W$ admits a unique projection $p_{\partial \Omega}(x)$ onto $\partial \Omega$;

(3) this projection satisfies

$$
p_{\partial \Omega}(x)=x-\frac{1}{2} \nabla b^{2}(x), \quad x \in W
$$

(4) it holds

$$
\nabla b(x)=N_{\partial \Omega}\left(p_{\partial \Omega}(x)\right), \quad x \in W .
$$

In particular, this latter property shows that $\nabla b(x)=N_{\partial \Omega}(x)$ for all $x \in \partial \Omega$ and $|\nabla b(x)|=1$ for all $x \in W$. Therefore, we define the extended unit normal by

$$
N(x):=\nabla b(x)=N_{\partial \Omega}\left(p_{\partial \Omega}(x)\right), \quad x \in W .
$$

2.2. Tangent vectors on $\partial \Omega$. For all $x \in \partial \Omega$, we denote by $T_{\partial \Omega}(x)$ the tangent plane to $\partial \Omega$ at $x$, that is, the orthogonal complement of $N_{\partial \Omega}(x)$. As $\partial \Omega$ is smooth, there exists a covering of $\partial \Omega$ by open balls $B_{1}, \ldots, B_{M}$ of $\mathbb{R}^{3}$ such that, for each index $k$, two smooth vector fields $\tau_{\partial \Omega}^{A}, \tau_{\partial \Omega}^{B}$ can be constructed on $\partial \Omega \cap B_{k}$ where, for all $x \in \partial \Omega \cap B_{k},\left(\tau_{\partial \Omega}^{A}(x), \tau_{\partial \Omega}^{B}(x)\right)$ is an orthonormal basis of $T_{\partial \Omega}(x)$. In all the sequel, the index $k$ will be implicitly considered as fixed and the restriction to $B_{k}$ will be omitted. In fact, for our needs, global properties and constructions will be easily obtained from local ones through a partition of unity subordinate to the covering.

Using that the Jacobian matrix $D N(x)=D^{2} b(x)$ of $N(x)$ is symmetric, differentiating the equality $|N(x)|^{2}=1$ entails

$$
\partial_{N} N(x)=D N(x) N(x)=0, \quad x \in W .
$$

In other words, $N(x)$ is an eigenvector of $D N(x)$ for the eigenvalue 0 . For all $x \in \partial \Omega$, the system $\left(\tau_{\partial \Omega}^{A}(x), \tau_{\partial \Omega}^{B}(x), N_{\partial \Omega}(x)\right)$ is an orthonormal basis of $\mathbb{R}^{3}$. In this basis, $D N(x)$ takes the form

$$
D N(x)=\left(\begin{array}{ccc}
\kappa_{\partial \Omega}^{A}(x) & \xi_{\partial \Omega}(x) & 0 \\
\xi_{\partial \Omega}(x) & \kappa_{\partial \Omega}^{B}(x) & 0 \\
0 & 0 & 0
\end{array}\right), \quad x \in \partial \Omega
$$


where $\kappa_{\partial \Omega}^{A}, \kappa_{\partial \Omega}^{B}$ and $\xi$ are smooth scalar fields defined on $\partial \Omega$.

If $R \in\{A, B\}$, we denote by $R^{*}$ the complementary index of $R$, that is, $R^{*}=B$ if $R=A$ and $R^{*}=A$ if $R=B$.

2.3. Extended tangent vectors. Let $d$ be defined in $W$ by

$$
d=\left(1+b \kappa_{\partial \Omega}^{A} \circ p_{\partial \Omega}\right)\left(1+b \kappa_{\partial \Omega}^{B} \circ p_{\partial \Omega}\right)-\left(b \xi_{\partial \Omega} \circ p_{\partial \Omega}\right)^{2} .
$$

Possibly adjusting $W$ so that $d(x)>0$ for all $x \in W$, we define in $W$ :

$$
\begin{aligned}
\quad \tau^{R}=\tau_{\partial \Omega}^{R} \circ p_{\partial \Omega}, \quad R=A, B, & \\
\kappa^{R} & =d^{-1}\left(\left(1+b \kappa_{\partial \Omega}^{R^{*}} \circ p_{\partial \Omega}\right)\left(\kappa_{\partial \Omega}^{R} \circ p_{\partial \Omega}\right)-b\left(\xi_{\partial \Omega} \circ p_{\partial \Omega}\right)^{2}\right), \quad R=A, B, \\
\xi & =d^{-1} \xi_{\partial \Omega} \circ p_{\partial \Omega}, \\
\kappa & =\kappa^{A}+\kappa^{B}, \\
\gamma^{R} & =\operatorname{div} \tau^{R}, \quad R=A, B .
\end{aligned}
$$

Obviously, for each $x \in W$, the triple $\left(\tau^{A}(x), \tau^{B}(x), N(x)\right)$ forms an orthonormal basis of $\mathbb{R}^{3}$. Next, we compute the normal and tangential derivatives of these vectors. We denote the tangential derivative $\partial_{\tau^{R}}$ by $\partial_{R}$ for simplicity. Specifically $\partial_{R} g(x)$ is the differential of the vector $g$ at $x$ in the direction $\tau^{R}$, viz.,

$$
\partial_{R} g(x):=D g(x) \tau^{R}(x) .
$$

Theorem 2.2. It holds in $W$ :

$$
\begin{aligned}
\partial_{N} \tau^{R} & =0, \\
\partial_{R} N & =\kappa^{R} \tau^{R}+\xi \tau^{R^{*}}, \\
\partial_{R} \tau^{R} & =-\kappa^{R} N-\gamma^{R^{*}} \tau^{R^{*}}, \\
\partial_{R^{*}} \tau^{R} & =\gamma^{R} \tau^{R^{*}}-\xi N, \\
\operatorname{div} N & =\operatorname{tr} D N=\Delta b=\kappa .
\end{aligned}
$$

Proof. Differentiating (2.1) in the direction $h \in \mathbb{R}^{3}$ and using (2.2) yields

$$
D p_{\partial \Omega}(x) h=h-(N(x) . h) N(x)-b(x) D N(x) h .
$$

Choosing $h=N(x)$ and using (2.3) entails $D p_{\partial \Omega}(x) N(x)=0$, then (2.6) in view of (2.5). Choosing now $h=\tau^{R}(x)$ gives

$$
D p_{\partial \Omega}(x) \tau^{R}(x)=\tau^{R}(x)-b(x) D N(x) \tau^{R}(x) .
$$

Differentiating $N(x)=N\left(p_{\partial \Omega}(x)\right)$ in the direction $\tau^{R}(x)$, one obtains using (2.11)

$$
\left(I+b(x) D N\left(p_{\partial \Omega}(x)\right)\right) \partial_{R} N(x)=D N\left(p_{\partial \Omega}(x)\right) \tau^{R}(x) .
$$

Plugging (2.4) into (2.12) yields (2.7). Differentiating the relations $\tau^{R}(x) \cdot N(x)=0,\left|\tau^{R}(x)\right|^{2}=$ 1 and $\tau^{R}(x) \cdot \tau^{R^{*}}(x)=0$ in the direction $\tau^{R}(x)$ yields $\partial_{R} \tau^{R}(x) \cdot N(x)=-\kappa^{R}(x), \partial_{R} \tau^{R}(x)$. $\tau^{R}(x)=0$ and $\partial_{R} \tau^{R}(x) \cdot \tau^{R^{*}}(x)+\partial_{R} \tau^{R^{*}}(x) \cdot \tau^{R}(x)=0$, respectively. From

$$
\operatorname{div} \tau^{R}(x)=\partial_{R} \tau^{R}(x) \cdot \tau^{R}(x)+\partial_{R^{*}} \tau^{R}(x) \cdot \tau^{R^{*}}(x)+\partial_{N} \tau^{R}(x) \cdot N(x)
$$

and the preceding relations one infers

$$
\gamma^{R}(x)=\partial_{R^{*}} \tau^{R}(x) \cdot \tau^{R^{*}}(x)=-\partial_{R^{*}} \tau^{R^{*}}(x) \cdot \tau^{R}(x) .
$$

This leads to (2.8). Differentiating $\tau^{R}(x) . N(x)=0$ and $\left|\tau^{R}(x)\right|^{2}=1$ in the direction $\tau^{R^{*}}(x)$ yields $\partial_{R^{*}} \tau^{R}(x) \cdot N(x)=-\xi(x)$ and $\partial_{R^{*}} \tau^{R}(x) \cdot \tau^{R}(x)=0$. With the help of (2.13) one arrives at (2.9). Finally, (2.10) is a straightforward consequence of (2.7) and the definitions.

Corollary 2.3. If $f$ is twice differentiable in $\Omega$ it holds

$$
\partial_{R} \partial_{N} f=\partial_{N} \partial_{R} f+\kappa^{R} \partial_{R} f+\xi \partial_{R^{*}} f .
$$


Proof. We have on one hand

$$
\begin{aligned}
\partial_{R} \partial_{N} f & =\partial_{R}(\nabla f \cdot N) \\
& =\partial_{R} \nabla f \cdot N+\nabla f \cdot\left(\kappa^{R} \tau^{R}+\xi \tau^{R^{*}}\right) \\
& =D^{2} f \tau^{R} \cdot N+\kappa^{R} \partial_{R} f+\xi \partial_{R^{*}} f,
\end{aligned}
$$

and on the other hand

$$
\partial_{N} \partial_{R} f=\partial_{N}\left(\nabla f \cdot \tau^{R}\right)=\partial_{N} \nabla f \cdot \tau^{R}=D^{2} f N \cdot \tau^{R}
$$

One concludes using the standard Schwarz lemma.

2.4. Divergence expression in the local basis. Let us decompose a $3 \times 3$ symmetric matrix $E$ in the local basis $\left(\tau^{A}, \tau^{B}, N\right)$ :

$$
E_{i j}=E_{N N} N_{i} N_{j}+\sum_{R=A, B} E_{N R}\left(N_{i} \tau_{j}^{R}+N_{j} \tau_{i}^{R}\right)+\sum_{R=A, B} E_{R R} \tau_{i}^{R} \tau_{j}^{R}+E_{A B}\left(\tau_{i}^{A} \tau_{j}^{B}+\tau_{j}^{A} \tau_{i}^{B}\right)
$$

Using Theorem 2.2 we obtain:

$$
\begin{aligned}
\partial_{j} E_{i j}= & \partial_{j} E_{N N} N_{i} N_{j}+E_{N N}\left(\partial_{N} N_{i}+\kappa N_{i}\right) \\
& +\sum_{R}\left[\partial_{j} E_{N R}\left(N_{i} \tau_{j}^{R}+N_{j} \tau_{i}^{R}\right)+E_{N R}\left(\partial_{R} N_{i}+N_{i} \partial_{j} \tau_{j}^{R}+\partial_{j} N_{j} \tau_{i}^{R}+\partial_{N} \tau_{i}^{R}\right)\right] \\
& +\sum_{R}\left[\partial_{j} E_{R R} \tau_{i}^{R} \tau_{j}^{R}+E_{R R}\left(\partial_{R} \tau_{i}^{R}+\tau_{i}^{R} \partial_{j} \tau_{j}^{R}\right)\right] \\
& +\partial_{j} E_{A B}\left(\tau_{i}^{A} \tau_{j}^{B}+\tau_{j}^{A} \tau_{i}^{B}\right)+E_{A B}\left(\partial_{B} \tau_{i}^{A}+\tau_{i}^{A} \partial_{j} \tau_{j}^{B}+\tau_{i}^{B} \partial_{j} \tau_{j}^{A}+\partial_{A} \tau_{i}^{B}\right) \\
= & \partial_{N} E_{N N} N_{i}+E_{N N} \kappa N_{i} \\
& +\sum_{R}\left[\partial_{R} E_{N R} N_{i}+\partial_{N} E_{N R} \tau_{i}^{R}+E_{N R}\left(\kappa^{R} \tau_{i}^{R}+\xi \tau_{i}^{R^{*}}+\gamma^{R} N_{i}+\kappa \tau_{i}^{R}\right)\right] \\
& +\sum_{R}\left[\partial_{R} E_{R R} \tau_{i}^{R}+E_{R R}\left(-\kappa^{R} N_{i}-\gamma^{R^{*}} \tau_{i}^{R^{*}}+\gamma^{R} \tau_{i}^{R}\right)\right] \\
& +\partial_{B} E_{A B} \tau_{i}^{A}+\partial_{A} E_{A B} \tau_{i}^{B}+E_{A B}\left(\gamma^{A} \tau_{i}^{B}-\xi N_{i}+\gamma^{B} \tau_{i}^{A}+\gamma^{A} \tau_{i}^{B}+\gamma^{B} \tau_{i}^{A}-\xi N_{i}\right) \\
= & N_{i}\left(\partial_{N} E_{N N}+\kappa E_{N N}+\sum_{R}\left(\partial_{R} E_{N R}+\gamma^{R} E_{N R}-\kappa^{R} E_{R R}\right)-2 \xi E_{A B}\right) \\
& +\sum_{R}\left[\tau _ { i } ^ { R } \left(\partial_{N} E_{N R}+\left(\kappa+\kappa^{R}\right) E_{N R}+\xi E_{N R^{*}}+\partial_{R} E_{R R}\right.\right. \\
& \left.\left.+\gamma^{R} E_{R R}-\gamma^{R} E_{R^{*} R^{*}}+\partial_{R^{*}} E_{A B}+2 \gamma^{R^{*}} E_{A B}\right)\right] .
\end{aligned}
$$

Hence

$$
\begin{gathered}
\operatorname{div} E=\left(\partial_{N} E_{N N}+\kappa E_{N N}+\sum_{R}\left(\partial_{R} E_{N R}+\gamma^{R} E_{N R}-\kappa^{R} E_{R R}\right)-2 \xi E_{A B}\right) N \\
+\sum_{R}\left(\partial_{N} E_{N R}+\left(\kappa+\kappa^{R}\right) E_{N R}+\xi E_{N R^{*}}+\partial_{R} E_{R R}\right. \\
\left.+\gamma^{R} E_{R R}-\gamma^{R} E_{R^{*} R^{*}}+\partial_{R^{*}} E_{A B}+2 \gamma^{R^{*}} E_{A B}\right) \tau^{R} .
\end{gathered}
$$




\section{FUnCTION SPACES}

3.1. Definitions and basic properties. Let $\Gamma_{0}$ be a subset of $\partial \Omega$ which is not everywhere flat and has nonzero 2-dimensional Hausdorff measure. Define

$$
\begin{aligned}
H_{\text {curl }}\left(\Omega, \mathbb{M}^{3}\right) & :=\left\{E \in L^{2}\left(\Omega, \mathbb{M}^{3}\right): \operatorname{Curl} E \in L^{2}\left(\Omega, \mathbb{M}^{3}\right)\right\}, \\
H_{\text {inc }}\left(\Omega, \mathbb{S}^{3}\right) & :=\left\{E \in L^{2}\left(\Omega, \mathbb{S}^{3}\right): \operatorname{inc} E \in L^{2}\left(\Omega, \mathbb{S}^{3}\right)\right\}, \\
\mathcal{H}(\Omega) & :=\left\{E \in H^{2}\left(\Omega, \mathbb{S}^{3}\right): \operatorname{div} E=0\right\}, \\
\mathcal{H}_{0}(\Omega) & :=\left\{E \in \mathcal{H}(\Omega): E=\left(\partial_{N} E \times N\right)^{t} \times N=0 \text { on } \partial \Omega\right\}, \\
\mathcal{H}_{\Gamma_{0}}(\Omega) & :=\left\{E \in \mathcal{H}(\Omega): E=\left(\partial_{N} E \times N\right)^{t} \times N=0 \text { on } \Gamma_{0}\right\}, \\
\tilde{H}_{0}^{1}\left(\Omega, \mathbb{R}^{3}\right) & :=\left\{u \in H_{0}^{1}\left(\Omega, \mathbb{R}^{3}\right): \int_{\Omega} u d x=0\right\}, \\
\tilde{H}^{3 / 2}\left(\partial \Omega, \mathbb{S}^{3}\right) & :=\left\{E \in H^{3 / 2}\left(\partial \Omega, \mathbb{S}^{3}\right): \int_{\partial \Omega} E N d S(x)=0\right\} .
\end{aligned}
$$

Given $\mathbb{E} \in \tilde{H}^{3 / 2}\left(\partial \Omega ; \mathbb{S}^{3}\right)$ and $\mathbb{F} \in H^{1 / 2}\left(\partial \Omega ; \mathbb{S}^{3}\right)$ such that $\int_{\partial \Omega} \mathbb{E} N d S(x)=0$ and $\mathbb{F} N=0$, we define the affine spaces

$$
\mathcal{H}_{\mathbb{E}, \mathbb{F}}(\Omega):=\left\{E \in \mathcal{H}(\Omega): E=\mathbb{E},\left(\partial_{N} E \times N\right)^{t} \times N=\mathbb{F} \text { on } \partial \Omega\right\},
$$

and

$$
\mathcal{H}_{\mathbb{E}, \mathbb{F} ; \Gamma_{0}}(\Omega):=\left\{E \in \mathcal{H}(\Omega): E=\mathbb{E},\left(\partial_{N} E \times N\right)^{t} \times N=\mathbb{F} \text { on } \Gamma_{0}\right\} .
$$

Obviously, in this latter case, it suffices that $\mathbb{E}$ and $\mathbb{F}$ be defined on $\Gamma_{0}$, and the condition $\int_{\partial \Omega} \mathbb{E} N d S(x)=0$ is not restrictive whenever $\Gamma_{0} \subset \subset \partial \Omega$. The spaces $\mathcal{H}(\Omega), \mathcal{H}_{0}(\Omega)$ and the above affine spaces are naturally endowed with the Hilbertian structure of $H^{2}\left(\Omega, \mathbb{S}^{3}\right)$.

Lemma 3.1. For all $E \in H^{2}\left(\Omega, \mathbb{S}^{3}\right)$ it holds in $W$

$$
\operatorname{Curl}^{\mathrm{t}} E \times N=-\left(\partial_{N} E \times N\right)^{t} \times N+\left(\sum_{R} \tau^{R} \times \partial_{R} E\right)^{t} \times N \quad \text { on } \partial \Omega .
$$

Proof. We compute componentwise

$$
\begin{aligned}
-\left[\operatorname{Curl}^{\mathrm{t}} E \times N\right]_{m q} & =\epsilon_{j q v} N_{v} \epsilon_{m l n} \partial_{l} E_{j n} \\
& =\epsilon_{j q v} N_{v} \epsilon_{m l n} N_{l} \partial_{N} E_{j n}+\epsilon_{j q v} N_{v} \epsilon_{m l n} \sum_{R} \tau_{l}^{R} \partial_{R} E_{j n} \\
& =\left(\left(\partial_{N} E \times N\right)^{t} \times N\right)_{m q}-\left(\left(\sum_{R} \tau^{R} \times \partial_{R} E\right)^{t} \times N\right)_{m q},
\end{aligned}
$$

proving the result.

Lemma 3.2. For all $V \in H^{1}\left(\Omega, \mathbb{R}^{3}\right)$ it holds in $W$

$$
\text { Curl } V \cdot N=\partial_{A} V_{B}-\partial_{B} V_{A}-\gamma^{B} V_{A}+\gamma^{A} V_{B} \text {. }
$$

Proof. We have

$$
\begin{aligned}
\operatorname{Curl} V . N= & \epsilon_{i j k} N_{i} \partial_{j} V_{k} \\
= & \epsilon_{i j k} N_{i}\left(\partial_{N} V_{k} N_{j}+\partial_{A} V_{k} \tau_{j}^{A}+\partial_{B} V_{k} \tau_{j}^{B}\right) \\
= & \partial_{A} V \cdot \tau^{B}-\partial_{B} V \cdot \tau^{A} \\
= & \partial_{A}\left(V_{A} \tau^{A}+V_{B} \tau^{B}+V_{N} N\right) \cdot \tau^{B}-\partial_{B}\left(V_{A} \tau^{A}+V_{B} \tau^{B}+V_{N} N\right) \cdot \tau^{A} \\
= & \partial_{A} V_{B}+\left(V_{A} \partial_{A} \tau^{A}+V_{B} \partial_{A} \tau^{B}+V_{N} \partial_{A} N\right) \cdot \tau^{B} \\
& -\partial_{B} V_{A}-\left(V_{A} \partial_{B} \tau^{A}+V_{B} \partial_{B} \tau^{B}+V_{N} \partial_{B} N\right) \cdot \tau^{A}
\end{aligned}
$$

Then one concludes using Theorem 2.2. 
Lemma 3.3. Every $E \in \mathcal{H}_{0}(\Omega)$ satisfies

$$
\begin{aligned}
\operatorname{div} \operatorname{Curl}^{\mathrm{t}} E & =0 & & \text { in } \Omega, \\
\mathrm{Curl}^{\mathrm{t}} E \times N & =0 & & \text { on } \partial \Omega, \\
\partial_{N} E & =0 & & \text { on } \partial \Omega .
\end{aligned}
$$

Proof. One has

$\left[\operatorname{div} \operatorname{Curl}^{\mathrm{t}} E\right]_{i}=\epsilon_{i k m} \partial_{j} \partial_{k} E_{j m}=\epsilon_{i k m} \partial_{k} \partial_{j} E_{m j}=0$

and $\mathrm{Curl}^{\mathrm{t}} E \times N=0$ by Lemma 3.1.

From (2.16) one infers on $\partial \Omega$

$$
\partial_{N} E_{N N}=0, \quad \partial_{N} E_{N R}=0 .
$$

Therefore (2.15) entails

$$
\partial_{N} E_{i j}=\sum_{R} \partial_{N} E_{R R} \tau_{i}^{R} \tau_{j}^{R}+\partial_{N} E_{A B}\left(\tau_{i}^{A} \tau_{j}^{B}+\tau_{j}^{A} \tau_{i}^{B}\right) .
$$

In the basis $\left(\tau^{A}, \tau^{B}, N\right)$ one has

$$
\begin{aligned}
\partial_{N} E & =\left(\begin{array}{ccc}
\partial_{N} E_{A A} & \partial_{N} E_{A B} & 0 \\
\partial_{N} E_{A B} & \partial_{N} E_{B B} & 0 \\
0 & 0 & 0
\end{array}\right), \quad \partial_{N} E \times N=\left(\begin{array}{ccc}
\partial_{N} E_{A B} & -\partial_{N} E_{A A} & 0 \\
\partial_{N} E_{B B} & -\partial_{N} E_{A B} & 0 \\
0 & 0 & 0
\end{array}\right) \\
0 & =\left(\partial_{N} E \times N\right)^{t} \times N=\left(\begin{array}{ccc}
\partial_{N} E_{B B} & -\partial_{N} E_{A B} & 0 \\
-\partial_{N} E_{A B} & \partial_{N} E_{A A} & 0 \\
0 & 0 & 0
\end{array}\right)
\end{aligned}
$$

whereby (3.5) follows.

Remark 3.4. In the same token, for a general symmetric tensor $T$, one has in the basis $\left(\tau^{A}, \tau^{B}, N\right)$ :

$$
\begin{gathered}
T=\left(\begin{array}{ccc}
T_{A A} & T_{A B} & T_{A N} \\
T_{B A} & T_{B B} & T_{B N} \\
T_{N A} & T_{N B} & T_{N N}
\end{array}\right), \quad T \times N=\left(\begin{array}{ccc}
T_{A B} & -T_{A A} & 0 \\
T_{B B} & -T_{B A} & 0 \\
T_{N B} & -T_{N A} & 0
\end{array}\right) \\
(T \times N)^{t} \times N=\left(\begin{array}{ccc}
T_{B B} & -T_{A B} & 0 \\
-T_{A B} & T_{A A} & 0 \\
0 & 0 & 0
\end{array}\right)
\end{gathered}
$$

Remark 3.5. Let $T=\operatorname{Curl}^{\mathrm{t}} E$ be such that $T \times N=0$ on $\partial \Omega$. Then, by Remark 3.4, one has $T_{Q R}=0$ for $R=A, B$ and $Q=A, B, N$. For fixed $i$, let $V_{j}=T_{i j}$ in Lemma 3.2. We infer $(\operatorname{Curl} T) N=(\operatorname{inc} E) N=0$ on $\partial \Omega$.

Lemma 3.6 (Kozono-Yanagisawa-von Wahl $[13,24])$. Let $F \in H_{\text {curl }}\left(\Omega ; \mathbb{M}^{3}\right)$ such that $\operatorname{div} F=$ 0 in $\Omega$ and $F \times N=0$ on $\partial \Omega$. Then $F \in H^{1}\left(\Omega, \mathbb{M}^{3}\right)$ and it holds

$$
\|\nabla F\|_{L^{2}(\Omega)} \leq C\|\operatorname{Curl} F\|_{L^{2}(\Omega)}
$$

for some positive constant $C$ independent of $F$.

Lemma 3.7. For all $E \in \mathcal{H}_{0}(\Omega)$ it holds

$$
\|E\|_{H^{2}(\Omega)} \leq C\left(\|E\|_{L^{2}(\Omega)}+\|\operatorname{Curl} E\|_{L^{2}(\Omega)}+\| \text { inc } E \|_{L^{2}(\Omega)}\right)
$$

for some positive constant $C$ independent of $E$.

Proof. By Lemma 3.6 we have already

$$
\|\nabla E\|_{L^{2}(\Omega)} \leq C\|\operatorname{Curl} E\|_{L^{2}(\Omega)} .
$$

Set $F=\operatorname{Curl}^{\mathrm{t}} E$. We have $\operatorname{Curl} F \in L^{2}(\Omega)$, and, by Lemma 3.3, $\operatorname{div} F=0$ in $\Omega$ and $F \times N=0$ on $\partial \Omega$. Hence Lemma 3.6 entails $\|\nabla F\|_{L^{2}(\Omega)} \leq C\|\operatorname{Curl} F\|_{L^{2}(\Omega)}$, i.e.,

$$
\left\|\partial_{i} \operatorname{Curl}^{\mathrm{t}} E\right\|_{L^{2}(\Omega)} \leq C \| \text { inc } E \|_{L^{2}(\Omega)} \text {. }
$$

This implies

$$
\left\|\operatorname{Curl} \partial_{i} E\right\|_{L^{2}(\Omega)} \leq C \| \text { inc } E \|_{L^{2}(\Omega)} \text {. }
$$


In addition, $\operatorname{div} \partial_{i} E=\partial_{i} \operatorname{div} E=0$ in $\Omega$. By Lemma $3.3, \partial_{N} E \times N=0$ on $\partial \Omega$, whereby, since $E=0$ on $\partial \Omega, \partial_{i} E \times N=0$ on $\partial \Omega$. Therefore, by Lemma 3.6,

$$
\left\|\partial_{j} \partial_{i} E\right\|_{L^{2}(\Omega)} \leq C\left\|\operatorname{Curl} \partial_{i} E\right\|_{L^{2}(\Omega)} .
$$

Using (3.10) we derive

$$
\left\|\partial_{j} \partial_{i} E\right\|_{L^{2}(\Omega)} \leq C \| \text { inc } E \|_{L^{2}(\Omega)},
$$

and the proof is achieved.

Theorem 3.8 (Poincaré). There exists a constant $C>0$ such that, for each $u \in H^{1}\left(\Omega, \mathbb{R}^{3}\right)$,

$$
\|u\|_{L^{2}(\Omega)} \leq C\left(\|\nabla u\|_{L^{2}(\Omega)}+\int_{\Gamma_{0}}|u \times N| d S\right) .
$$

Proof. By contradiction, assume that for each $k \in \mathbb{N}$, there exists a $u_{k} \in H^{1}\left(\Omega ; \mathbb{R}^{3}\right)$ such that

$$
\left\|u_{k}\right\|_{L^{2}(\Omega)}>k\left(\left\|\nabla u_{k}\right\|_{L^{2}(\Omega)}+\int_{\Gamma_{0}}\left|u_{k} \times N\right| d S\right) .
$$

Defining $\dot{u}_{k}:=u_{k} /\left\|u_{k}\right\|_{L^{2}(\Omega)}$, one has $\left\|\dot{u}_{k}\right\|_{L^{2}(\Omega)}=1$ and hence (i) $\left\|\nabla \dot{u}_{k}\right\|_{L^{2}(\Omega)} \rightarrow 0$, (ii) $\int_{\Gamma_{0}}\left|\dot{u}_{k} \times N\right| d S \rightarrow 0$ as $k \rightarrow \infty$. By (i) and Rellich's theorem there exists $v \in H^{s}\left(\Omega, \mathbb{R}^{3}\right)$, $1 / 2<s<1$, such that a nonrelabelled subsequence $\dot{u}_{k} \rightarrow v$ in $H^{s}\left(\Omega, \mathbb{R}^{3}\right)$, and hence by virtue of (i) and for every $\varphi \in \mathcal{D}\left(\Omega, \mathbb{R}^{3}\right)$,

$$
\int_{\Omega} v \operatorname{div} \varphi d x=\lim _{k \rightarrow \infty} \int_{\Omega} \dot{u}_{k} \operatorname{div} \varphi d x=-\lim _{k \rightarrow \infty} \int_{\Omega} D \dot{u}_{k} \varphi d x=0,
$$

whereby $\nabla v=0$, meaning that $v$ is a constant vector. Condition (ii) now implies that $\int_{\Gamma_{0}} \mid \dot{u}_{k} \times$ $N\left|d S \rightarrow \int_{\Gamma_{0}}\right| v \times N \mid d S=0$ as $k \rightarrow \infty$, i.e., $v \times N=0$ and thus $v$ is parallel to $N$, which is not constant and of unit length, and hence $v=0$, a contradiction, since $\|v\|_{L^{2}(\Omega)}=1$.

Theorem 3.9 (Coercivity). There exists a positive constant $C$ such that, for each $E \in \mathcal{H}_{0}(\Omega)$,

$$
\|E\|_{H^{2}(\Omega)} \leq C \| \text { inc } E \|_{L^{2}(\Omega)} .
$$

Proof. By Theorem 3.8, the tensor counterpart of (3.12) reads

$$
\|F\|_{L^{2}(\Omega)} \leq C\left(\|\nabla F\|_{L^{2}(\Omega)}+\int_{\Gamma_{0}}|F \times N| d S\right),
$$

for all $F \in H^{1}\left(\Omega, \mathbb{M}^{3}\right)$. By Lemma 3.3, div $\operatorname{Curl}^{\mathrm{t}} E=0$ in $\Omega$ and $\operatorname{Curl}^{\mathrm{t}} E \times N=0$ on $\partial \Omega$. Hence by Lemma 3.6 and again by Theorem 3.8, one has (with the nonrelabeled constant $C>0)$,

$$
\begin{aligned}
&\|E\|_{L^{2}(\Omega)} \leq C\|\nabla E\|_{L^{2}(\Omega)} \leq C\|\operatorname{Curl} E\|_{L^{2}(\Omega)} \\
& \leq C\left\|\operatorname{Curl}^{\mathrm{t}} E\right\|_{L^{2}(\Omega)} \\
& \leq C\left\|\nabla \operatorname{Curl}^{\mathrm{t}} E\right\|_{L^{2}(\Omega)} \leq C\left\|\operatorname{Curl~Curl}^{\mathrm{t}} E\right\|_{L^{2}(\Omega)}=C \| \text { inc } E \|_{L^{2}(\Omega)} .
\end{aligned}
$$

The proof is completed using Lemma 3.7 .

\subsection{Lifting of boundary traces.}

Theorem 3.10. Let $g \in \tilde{H}_{0}^{1}\left(\Omega, \mathbb{R}^{3}\right)$. There exists $U \in H_{0}^{2}\left(\Omega, \mathbb{S}^{3}\right)$ such that $\operatorname{div} U=g$.

Proof. Step 1. Let $v \in H^{1}\left(\Omega, \mathbb{R}^{3}\right)$ be a solution (unique up to a rigid motion) of

$$
\begin{cases}-\operatorname{div} \nabla^{s} v=g & \text { in } \Omega \\ \nabla^{s} v N=0 & \text { on } \partial \Omega\end{cases}
$$

and set $V=\nabla^{s} v$. By elliptic regularity, $v \in H^{3}(\Omega)$, thus $V \in H^{2}(\Omega)$. We have $\operatorname{div} V=g$ in $\Omega$ and $V N=0$ on $\partial \Omega$.

Step 2. We aim at defining $U=V+W$ where $W=$ inc $\Psi, \Psi \in H^{4}\left(\Omega, \mathbb{S}^{3}\right)$, must satisfy:

$$
\begin{aligned}
W N & =0 \quad \text { on } \partial \Omega, \\
W \tau^{R} & =-V \tau^{R} \quad \text { on } \partial \Omega, \\
\partial_{N} W & =-\partial_{N} V \quad \text { on } \partial \Omega .
\end{aligned}
$$


We assume a priori that $\Psi \in H^{4}\left(\Omega, \mathbb{S}^{3}\right)$ satisfies

$$
\Psi=\partial_{N} \Psi=0 \quad \text { on } \quad \partial \Omega .
$$

Then (3.14) holds true by Remark 3.5 and Lemma 3.1. We are going to derive other conditions on $\Psi$ such that (3.15)-(3.16) are also satisfied.

Step 3. Let us rewrite the traces of $V$ and $\partial_{N} V$ on $\partial \Omega$ in the local basis $\left(\tau^{A}, \tau^{B}, N\right)$ as

$$
V=\left(\begin{array}{ccc}
V_{A A} & V_{A B} & V_{A N} \\
V_{A B} & V_{B B} & V_{B N} \\
V_{A N} & V_{B N} & V_{N N}
\end{array}\right)=\left(\begin{array}{ccc}
V_{A A} & V_{A B} & 0 \\
V_{A B} & V_{B B} & 0 \\
0 & 0 & 0
\end{array}\right), \partial_{N} V=\left(\begin{array}{lll}
\partial_{N} V_{A A} & \partial_{N} V_{A B} & \partial_{N} V_{A N} \\
\partial_{N} V_{A B} & \partial_{N} V_{B B} & \partial_{N} V_{B N} \\
\partial_{N} V_{A N} & \partial_{N} V_{B N} & \partial_{N} V_{N N}
\end{array}\right) .
$$

Assume that

$$
\partial_{N}^{2} \Psi=\left(\begin{array}{ccc}
-V_{B B} & V_{A B} & 0 \\
V_{A B} & -V_{A A} & 0 \\
0 & 0 & 0
\end{array}\right)
$$

and

$$
\partial_{N}^{3} \Psi=\left(\begin{array}{ccc}
\left(-\partial_{N}+2 \kappa^{A}\right) V_{B B}-2 \xi V_{A B} & \left(\partial_{N}-\kappa\right) V_{A B}+\xi\left(V_{A A}+V_{B B}\right) & 0 \\
\left(\partial_{N}-\kappa\right) V_{A B}+\xi\left(V_{A A}+V_{B B}\right) & \left(-\partial_{N}+2 \kappa^{B}\right) V_{A A}-2 \xi V_{A B} & 0 \\
0 & 0 & 0
\end{array}\right) .
$$

Let us compute the components of the vector $W \tau^{R}$.

- For $W \tau^{R} \cdot N$, it holds

$$
W \tau^{R} \cdot N: W \tau^{R} \cdot N=W N \cdot \tau^{R}=0=-V_{R N} .
$$

- For $W \tau^{R} \cdot \tau^{R}$, we compute componentwise

$$
\begin{aligned}
W_{i j} \tau_{j}^{A} \tau_{i}^{A} & =\epsilon_{i k m} \tau_{i}^{A} \epsilon_{j l n} \tau_{j}^{A} \partial_{k} \partial_{l} \Psi_{m n} \\
& =\epsilon_{i k m} \tau_{i}^{A} \epsilon_{j l n} \partial_{k}\left(\tau_{j}^{A} \partial_{l} \Psi_{m n}\right)-\epsilon_{i k m} \tau_{i}^{A} \epsilon_{j l n}\left(\partial_{k} \tau_{j}^{A}\right) \partial_{l} \Psi_{m n} .
\end{aligned}
$$

The last term of the right hand side vanishes by (3.17), hence

$$
\begin{aligned}
W_{i j} \tau_{j}^{A} \tau_{i}^{A} & =\epsilon_{i k m} \tau_{i}^{A} \partial_{k}\left(\epsilon_{j l n} \tau_{j}^{A} \partial_{l} \Psi_{m n}\right) \\
& =\epsilon_{i k m} \tau_{i}^{A}\left(\tau_{k}^{B} \partial_{B}+N_{k} \partial_{N}\right)\left(\epsilon_{j l n} \tau_{j}^{A}\left(\tau_{l}^{B} \partial_{B}+N_{l} \partial_{N}\right) \Psi_{m n}\right) \\
& =\left(N_{m} \partial_{B}-\tau_{m}^{B} \partial_{N}\right)\left(N_{n} \partial_{B}-\tau_{n}^{B} \partial_{N}\right) \Psi_{m n} \\
& =N_{m} \partial_{B}\left(N_{n} \partial_{B} \Psi_{m n}\right)-N_{m} \partial_{B}\left(\tau_{n}^{B} \partial_{N} \Psi_{m n}\right)-\tau_{m}^{B} \partial_{N}\left(N_{n} \partial_{B} \Psi_{m n}\right)+\tau_{m}^{B} \partial_{N}\left(\tau_{n}^{B} \partial_{N} \Psi_{m n}\right),
\end{aligned}
$$

which again by (3.17) yields

$$
W_{i j} \tau_{j}^{A} \tau_{i}^{A}=-\tau_{m}^{B} N_{n} \partial_{N}\left(\partial_{B} \Psi_{m n}\right)+\tau_{m}^{B} \tau_{n}^{B} \partial_{N}\left(\partial_{N} \Psi_{m n}\right),
$$

that is, by (3.17) and (2.14),

$$
W_{i j} \tau_{j}^{A} \tau_{i}^{A}=\partial_{N} \partial_{N}\left(\tau_{m}^{B} \tau_{n}^{B} \Psi_{m n}\right)=\partial_{N}^{2} \Psi_{B B} .
$$

Similarly, it holds $W_{i j} \tau_{j}^{B} \tau_{i}^{B}=\partial_{N}^{2} \Psi_{A A}$.

- Now, consider $W \tau^{R} \cdot \tau^{R^{*}}$ and compute componentwise

$$
\begin{aligned}
W_{i j} \tau_{j}^{A} \tau_{i}^{B} & =\epsilon_{i k m} \tau_{i}^{B} \epsilon_{j l n} \tau_{j}^{A} \partial_{k} \partial_{l} \Psi_{m n}=\epsilon_{i k m} \tau_{i}^{B} \partial_{k}\left(\epsilon_{j l n} \tau_{j}^{A} \partial_{l} \Psi_{m n}\right) \\
& =\epsilon_{i k m} \tau_{i}^{B}\left(\tau_{k}^{A} \partial_{A}+N_{k} \partial_{N}\right)\left(\epsilon_{j l n} \tau_{j}^{A}\left(\tau_{l}^{B} \partial_{B}+N_{l} \partial_{N}\right) \Psi_{m n}\right) \\
& =\left(-N_{m} \partial_{A}+\tau_{m}^{A} \partial_{N}\right)\left(N_{n} \partial_{B}-\tau_{n}^{B} \partial_{N}\right) \Psi_{m n} \\
& =-N_{m} \partial_{A}\left(N_{n} \partial_{B} \Psi_{m n}\right)+N_{m} \partial_{A}\left(\tau_{n}^{B} \partial_{N} \Psi_{m n}\right)+\tau_{m}^{A} \partial_{N}\left(N_{n} \partial_{B} \Psi_{m n}\right)-\tau_{m}^{A} \partial_{N}\left(\tau_{n}^{B} \partial_{N} \Psi_{m n}\right) .
\end{aligned}
$$

By (3.17) and (2.14), this reads

$$
W_{i j} \tau_{j}^{A} \tau_{i}^{B}=-\partial_{N}^{2} \Psi_{A B}
$$

Thus (3.15) is satisfied by (3.18) and (3.20)-(3.22).

Step 4. Let us compute $\partial_{N} W$.

- We first compute $\partial_{N} W \tau^{R} \cdot \tau^{R}$. Recall that, from Corollary 2.3, one has

$$
\partial_{N A}=\partial_{A N}-\kappa^{A} \partial_{A}-\xi \partial_{B} .
$$


By mere projections, we have

$$
\begin{aligned}
\partial_{N} W_{i j} \tau_{j}^{A} \tau_{i}^{A}= & \partial_{N}\left[\epsilon_{i k m} \epsilon_{j l n} \tau_{i}^{A} \tau_{j}^{A} \partial_{k} \partial_{l} \Psi_{m n}\right] \\
= & \partial_{N}\left[\epsilon_{i k m} \epsilon_{j l n} \tau_{i}^{A} \tau_{j}^{A}\left(\tau_{k}^{A} \partial_{A}+\tau_{k}^{B} \partial_{B}+N_{k} \partial_{N}\right)\left(\tau_{l}^{A} \partial_{A}+\tau_{l}^{B} \partial_{B}+N_{l} \partial_{N}\right) \Psi_{m n}\right] \\
= & \partial_{N}\left[\epsilon_{i k m} \tau_{i}^{A}\left(\tau_{k}^{A} \partial_{A}+\tau_{k}^{B} \partial_{B}+N_{k} \partial_{N}\right)\left(\epsilon_{j l n} \tau_{j}^{A}\left(\tau_{l}^{A} \partial_{A}+\tau_{l}^{B} \partial_{B}+N_{l} \partial_{N}\right) \Psi_{m n}\right)\right. \\
& \left.-\epsilon_{i k m} \epsilon_{j l n} \tau_{i}^{A}\left(\tau_{k}^{A} \partial_{A}+\tau_{k}^{B} \partial_{B}+N_{k} \partial_{N}\right) \tau_{j}^{A}\left(\tau_{l}^{A} \partial_{A}+\tau_{l}^{B} \partial_{B}+N_{l} \partial_{N}\right) \Psi_{m n}\right] \\
= & \partial_{N}\left[\left(N_{m} \partial_{B}-\tau_{m}^{B} \partial_{N}\right)\left(N_{n} \partial_{B}-\tau_{n}^{B} \partial_{N}\right) \Psi_{m n}\right. \\
& \left.-\epsilon_{j l n}\left(\left(N_{m} \partial_{B}-\tau_{m}^{B} \partial_{N}\right) \tau_{j}^{A}\right)\left(\tau_{l}^{A} \partial_{A}+\tau_{l}^{B} \partial_{B}+N_{l} \partial_{N}\right) \Psi_{m n}\right] \\
= & \left(N_{m} \partial_{N B}-\tau_{m}^{B} \partial_{N N}\right)\left(N_{n} \partial_{B}-\tau_{n}^{B} \partial_{N}\right) \Psi_{m n} \\
& -\epsilon_{j l n} N_{m} \partial_{B} \tau_{j}^{A}\left(\tau_{l}^{A} \partial_{N A}+\tau_{l}^{B} \partial_{N B}+N_{l} \partial_{N N}\right) \Psi_{m n} \\
& -\epsilon_{j l n}\left(\left(N_{m} \partial_{N B}-\tau_{m}^{B} \partial_{N N}\right) \tau_{j}^{A}\right)\left(\tau_{l}^{A} \partial_{A}+\tau_{l}^{B} \partial_{B}+N_{l} \partial_{N}\right) \Psi_{m n},
\end{aligned}
$$

and hence from (3.17) and (3.23), the right hand side of (3.24) equals to

$$
\left(N_{m} \partial_{N B}-\tau_{m}^{B} \partial_{N N}\right)\left(N_{n} \partial_{B}-\tau_{n}^{B} \partial_{N}\right) \Psi_{m n}-\epsilon_{j l n} N_{m} \partial_{B} \tau_{j}^{A}\left(\tau_{l}^{A} \partial_{N A}+\tau_{l}^{B} \partial_{N B}+N_{l} \partial_{N N}\right) \Psi_{m n} .
$$

By (3.23), it follows that

$$
\begin{aligned}
\partial_{N} W_{i j} \tau_{j}^{A} \tau_{i}^{A}=\left(N_{m} \partial_{B N}-\kappa^{B} N_{m} \partial_{B}-\xi N_{m} \partial_{A}-\tau_{m}^{B} \partial_{N N}\right)\left(N_{n} \partial_{B}-\tau_{n}^{B} \partial_{N}\right) \Psi_{m n} \\
-\epsilon_{j l n} N_{m}\left(\gamma^{A} \tau_{j}^{B}-\xi N_{j}\right)\left(\tau_{l}^{A} \partial_{N A}+\tau_{l}^{B} \partial_{N B}+N_{l} \partial_{N N}\right) \Psi_{m n},
\end{aligned}
$$

thus by virtue of (3.17) and (3.23),

$$
\begin{aligned}
\partial_{N} W_{i j} \tau_{j}^{A} \tau_{i}^{A} & =\left(N_{m} \partial_{B N}-\tau_{m}^{B} \partial_{N N}\right)\left(N_{n} \partial_{B}-\tau_{n}^{B} \partial_{N}\right) \Psi_{m n}-\epsilon_{j l n} N_{m}\left(\gamma^{A} \tau_{j}^{B}-\xi N_{j}\right)\left(N_{l} \partial_{N N}\right) \Psi_{m n} \\
& =\left(N_{m} \partial_{B}-\tau_{m}^{B} \partial_{N}\right)\left(N_{n} \partial_{N B}-\tau_{n}^{B} \partial_{N N}\right) \Psi_{m n}-N_{m}\left(\gamma^{A} \tau_{n}^{A}\right) \partial_{N N} \Psi_{m n},
\end{aligned}
$$

and again by (3.23),

$$
\begin{aligned}
& \partial_{N} W_{i j} \tau_{j}^{A} \tau_{i}^{A}=\left(N_{m} \partial_{B}-\tau_{m}^{B} \partial_{N}\right)\left(N_{n} \partial_{B N}-\kappa^{B} N_{n} \partial_{B}-\xi N_{n} \partial_{A}-\tau_{n}^{B} \partial_{N N}\right) \Psi_{m n} \\
& -\gamma^{A} N_{m} \tau_{n}^{A} \partial_{N N} \Psi_{m n} .
\end{aligned}
$$

By (3.17), this entails that

$$
\begin{array}{r}
\partial_{N} W_{i j} \tau_{j}^{A} \tau_{i}^{A}=N_{m} \partial_{B}\left(-\tau_{n}^{B} \partial_{N N}\right) \Psi_{m n}-\tau_{m}^{B}\left(N_{n} \partial_{N B N}-\kappa^{B} N_{n} \partial_{N B}-\left(\partial_{N} \kappa^{B}\right) N_{n} \partial_{B}\right. \\
\left.-\xi N_{n} \partial_{N A}-\left(\partial_{N} \xi\right) N_{n} \partial_{A}-\tau_{n}^{B} \partial_{N N N}\right) \Psi_{m n}-\gamma^{A} N_{m} \tau_{n}^{A} \partial_{N N} \Psi_{m n},
\end{array}
$$

whereby, using again (3.17) and (3.23),

$$
\begin{aligned}
\partial_{N} W_{i j} \tau_{j}^{A} \tau_{i}^{A}= & -N_{m} \partial_{B}\left(\tau_{n}^{B} \partial_{N N}\right) \Psi_{m n}-\tau_{m}^{B}\left(N_{n} \partial_{N B N}-\tau_{n}^{B} \partial_{N N N}\right) \Psi_{m n}-\gamma^{A} N_{m} \tau_{n}^{A} \partial_{N N} \Psi_{m n} \\
= & \left.-N_{m}\left(-\kappa^{B} N_{n} \partial_{N N}-\gamma^{A} \tau_{n}^{A} \partial_{N N}+\tau_{n}^{B} \partial_{B N N}\right)\right) \Psi_{m n} \\
& -\tau_{m}^{B}\left(N_{n} \partial_{B N N}-\tau_{n}^{B} \partial_{N N N}\right) \Psi_{m n}-\gamma^{A} N_{m} \tau_{n}^{A} \partial_{N N} \Psi_{m n} .
\end{aligned}
$$

Therefore,

$$
\begin{aligned}
\partial_{N} W_{i j} \tau_{j}^{A} \tau_{i}^{A} & =\kappa^{B} N_{m} N_{n} \partial_{N N} \Psi_{m n}-\left(N_{m} \tau_{n}^{B}+N_{n} \tau_{m}^{B}\right) \partial_{B N N} \Psi_{m n}+\tau_{m}^{B} \tau_{n}^{B} \partial_{N N N} \Psi_{m n} \\
& =\kappa^{B} N_{m} N_{n} \partial_{N N} \Psi_{m n}-2 N_{m} \tau_{n}^{B} \partial_{B N N} \Psi_{m n}+\tau_{m}^{B} \tau_{n}^{B} \partial_{N N N} \Psi_{m n}
\end{aligned}
$$

Yet

$$
\begin{aligned}
\partial_{B}\left(N_{m} \tau_{n}^{B}\right) & =\left(\kappa^{B} \tau_{m}^{B}+\xi \tau_{m}^{A}\right) \tau_{n}^{B}+N_{m}\left(-\kappa^{B} N_{n}-\gamma^{A} \tau_{n}^{A}\right) \\
& =\kappa^{B} \tau_{m}^{B} \tau_{n}^{B}+\xi \tau_{m}^{A} \tau_{n}^{B}-\kappa^{B} N_{m} N_{n}-\gamma^{A} N_{m} \tau_{n}^{A}
\end{aligned}
$$


This yields

$$
\begin{aligned}
\partial_{N} W_{i j} \tau_{j}^{A} \tau_{i}^{A}= & \kappa^{B} N_{m} N_{n} \partial_{N N} \Psi_{m n}-2 \partial_{B}\left(N_{m} \tau_{n}^{B} \partial_{N N} \Psi_{m n}\right) \\
& +2\left(\kappa^{B} \tau_{m}^{B} \tau_{n}^{B}+\xi \tau_{m}^{A} \tau_{n}^{B}-\kappa^{B} N_{m} N_{n}-\gamma^{A} N_{m} \tau_{n}^{A}\right) \partial_{N N} \Psi_{m n}+\tau_{m}^{B} \tau_{n}^{B} \partial_{N N N} \Psi_{m n} \\
= & -\kappa^{B} N_{m} N_{n} \partial_{N N} \Psi_{m n}-2 \partial_{B}\left(N_{m} \tau_{n}^{B} \partial_{N N} \Psi_{m n}\right) \\
& +2\left(\kappa^{B} \tau_{m}^{B} \tau_{n}^{B}+\xi \tau_{m}^{A} \tau_{n}^{B}-\gamma^{A} N_{m} \tau_{n}^{A}\right) \partial_{N N} \Psi_{m n}+\tau_{m}^{B} \tau_{n}^{B} \partial_{N N N} \Psi_{m n} \\
= & -\kappa^{B} \partial_{N N} \Psi_{N N}-2 \partial_{B} \partial_{N N} \Psi_{B N} \\
& +2 \kappa^{B} \partial_{N N} \Psi_{B B}+2 \xi \partial_{N N} \Psi_{A B}-2 \gamma^{A} \partial_{N N} \Psi_{A N}+\partial_{N N N} \Psi_{B B},
\end{aligned}
$$

which implies by (3.18) and (3.19) that

$$
\partial_{N} W_{i j} \tau_{j}^{A} \tau_{i}^{A}=-2 \kappa^{B} V_{A A}+2 \xi V_{A B}+\partial_{N}^{3} \Psi_{B B}=-\partial_{N} V_{A A} .
$$

We have obtained $\partial_{N} W_{A A}=-\partial_{N} V_{A A}$. Similarly we find $\partial_{N} W_{B B}=-\partial_{N} V_{B B}$.

- Then we compute $\partial_{N} W \tau^{R} \cdot \tau^{R^{*}}$. We have

$$
\begin{aligned}
\partial_{N} W_{i j} \tau_{j}^{A} \tau_{i}^{B}= & \partial_{N}\left[\epsilon_{i k m} \epsilon_{j l n} \tau_{i}^{B} \tau_{j}^{A} \partial_{k} \partial_{l} \Psi_{m n}\right] \\
= & \partial_{N}\left[\epsilon_{i k m} \epsilon_{j l n} \tau_{i}^{B} \tau_{j}^{A}\left(\tau_{k}^{A} \partial_{A}+\tau_{k}^{B} \partial_{B}+N_{k} \partial_{N}\right)\left(\tau_{l}^{A} \partial_{A}+\tau_{l}^{B} \partial_{B}+N_{l} \partial_{N}\right) \Psi_{m n}\right] \\
= & \partial_{N}\left[\left(-N_{m} \partial_{A}+\tau_{m}^{A} \partial_{N}\right)\left(N_{n} \partial_{B}-\tau_{n}^{B} \partial_{N}\right) \Psi_{m n}\right. \\
& \left.-\epsilon_{j l n}\left(-N_{m} \partial_{A}+\tau_{m}^{A} \partial_{N}\right) \tau_{j}^{A}\left(\tau_{l}^{A} \partial_{A}+\tau_{l}^{B} \partial_{B}+N_{l} \partial_{N}\right) \Psi_{m n}\right],
\end{aligned}
$$

which by virtue (3.17) rewrites as

$$
\begin{aligned}
\partial_{N} W_{i j} \tau_{j}^{A} \tau_{i}^{B}=\left(-N_{m} \partial_{N A}+\right. & \left.\tau_{m}^{A} \partial_{N N}\right)\left(N_{n} \partial_{B}-\tau_{n}^{B} \partial_{N}\right) \Psi_{m n} \\
& +\epsilon_{j l n} N_{m}\left(-\kappa^{A} N_{j}-\gamma^{B} \tau_{j}^{B}\right)\left(\tau_{l}^{A} \partial_{N A}+\tau_{l}^{B} \partial_{N B}+N_{l} \partial_{N N}\right) \Psi_{m n} .
\end{aligned}
$$

Hence, by (3.17) and (3.23),

$$
\begin{aligned}
\partial_{N} W_{i j} \tau_{j}^{A} \tau_{i}^{B}=\left(-N_{m} \partial_{A N}+\right. & \left.\kappa^{A} N_{m} \partial_{A}+\xi N_{m} \partial_{B}+\tau_{m}^{A} \partial_{N N}\right)\left(N_{n} \partial_{B}-\tau_{n}^{B} \partial_{N}\right) \Psi_{m n} \\
& +\epsilon_{j l n} N_{m}\left(-\kappa^{A} N_{j}-\gamma^{B} \tau_{j}^{B}\right)\left(\tau_{l}^{A} \partial_{N A}+\tau_{l}^{B} \partial_{N B}+N_{l} \partial_{N N}\right) \Psi_{m n}
\end{aligned}
$$

and by (3.17) again, one has

$$
\begin{aligned}
\partial_{N} W_{i j} \tau_{j}^{A} \tau_{i}^{B} & =\left(-N_{m} \partial_{A N}+\tau_{m}^{A} \partial_{N N}\right)\left(N_{n} \partial_{B}-\tau_{n}^{B} \partial_{N}\right) \Psi_{m n}-\epsilon_{j l n} N_{m}\left(\kappa^{A} N_{j}+\gamma^{B} \tau_{j}^{B}\right) N_{l} \partial_{N N} \Psi_{m n} \\
& =\left(-N_{m} \partial_{A}+\tau_{m}^{A} \partial_{N}\right)\left(N_{n} \partial_{N B}-\tau_{n}^{B} \partial_{N N}\right) \Psi_{m n}-\gamma^{B} N_{m} \tau_{n}^{A} \partial_{N N} \Psi_{m n}
\end{aligned}
$$

Again by (3.23), the right hand side of (3.28) equals to

$$
\left(-N_{m} \partial_{A}+\tau_{m}^{A} \partial_{N}\right)\left(N_{n} \partial_{B N}-\kappa^{B} N_{n} \partial_{B}-\xi N_{n} \partial_{A}-\tau_{n}^{B} \partial_{N N}\right) \Psi_{m n}-\gamma^{B} N_{m} \tau_{n}^{A} \partial_{N N} \Psi_{m n},
$$

which by (3.17) is rewritten as

$N_{m} \partial_{A} \tau_{n}^{B} \partial_{N N} \psi_{m n}+\tau_{m}^{A}\left(N_{n} \partial_{N B N}-\kappa^{B} N_{n} \partial_{N B}-\xi N_{n} \partial_{N A}-\tau_{n}^{B} \partial_{N N N}\right) \Psi_{m n}-\gamma^{B} N_{m} \tau_{n}^{A} \partial_{N N} \Psi_{m n}$.

Therefore, (3.17) and (3.23) imply that

$$
\begin{aligned}
\partial_{N} W_{i j} \tau_{j}^{A} \tau_{i}^{B}= & N_{m} \partial_{A}\left(\tau_{n}^{B} \partial_{N N}\right) \psi_{m n}+\tau_{m}^{A}\left(N_{n} \partial_{N B N}-\tau_{n}^{B} \partial_{N N N}\right) \Psi_{m n}-\gamma^{B} N_{m} \tau_{n}^{A} \partial_{N N} \Psi_{m n} \\
= & \left.N_{m}\left(\gamma^{B} \tau_{n}^{A} \partial_{N N}-\xi N_{n} \partial_{N N}+\tau_{n}^{B} \partial_{A N N}\right)\right) \Psi_{m n} \\
& +\tau_{m}^{A}\left(N_{n} \partial_{B N N}-\tau_{n}^{B} \partial_{N N N}\right) \Psi_{m n}-\gamma^{B} N_{m} \tau_{n}^{A} \partial_{N N} \Psi_{m n} \\
= & -\xi N_{m} N_{n} \partial_{N N} \Psi_{m n}+N_{m} \tau_{n}^{B} \partial_{A N N} \Psi_{m n}+N_{n} \tau_{m}^{A} \partial_{B N N} \Psi_{m n}-\tau_{m}^{A} \tau_{n}^{B} \partial_{N N N} \Psi_{m n} .
\end{aligned}
$$

Yet,

$\partial_{A}\left(N_{m} \tau_{n}^{B}\right)+\partial_{B}\left(N_{n} \tau_{m}^{A}\right)=\kappa \tau_{m}^{A} \tau_{n}^{B}+\xi\left(\tau_{m}^{A} \tau_{n}^{A}+\tau_{m}^{B} \tau_{n}^{B}\right)+\gamma^{B} N_{m} \tau_{n}^{A}+\gamma^{A} N_{n} \tau_{m}^{B}-2 \xi N_{m} N_{n}$. 
This yields

$$
\begin{aligned}
\partial_{N} W_{i j} \tau_{j}^{A} \tau_{i}^{B}= & -\xi N_{m} N_{n} \partial_{N N} \Psi_{m n}+\partial_{A}\left(N_{m} \tau_{n}^{B} \partial_{N N} \Psi_{m n}\right)+\partial_{B}\left(N_{n} \tau_{m}^{A} \partial_{N N} \Psi_{m n}\right) \\
& -\left(\kappa \tau_{m}^{A} \tau_{n}^{B}+\xi\left(\tau_{m}^{A} \tau_{n}^{A}+\tau_{m}^{B} \tau_{n}^{B}\right)+\gamma^{B} N_{m} \tau_{n}^{A}+\gamma^{A} N_{n} \tau_{m}^{B}-2 \xi N_{m} N_{n}\right) \partial_{N N} \Psi_{m n} \\
& -\tau_{m}^{A} \tau_{n}^{B} \partial_{N N N} \Psi_{m n} \\
= & \xi N_{m} N_{n} \partial_{N N} \Psi_{m n}+\partial_{A}\left(N_{m} \tau_{n}^{B} \partial_{N N} \Psi_{m n}\right)+\partial_{B}\left(N_{n} \tau_{m}^{A} \partial_{N N} \Psi_{m n}\right) \\
& -\left(\kappa \tau_{m}^{A} \tau_{n}^{B}+\xi\left(\tau_{m}^{A} \tau_{n}^{A}+\tau_{m}^{B} \tau_{n}^{B}\right)+\gamma^{B} N_{m} \tau_{n}^{A}+\gamma^{A} N_{n} \tau_{m}^{B}\right) \partial_{N N} \Psi_{m n}-\tau_{m}^{A} \tau_{n}^{B} \partial_{N N N} \Psi_{m n} \\
= & \xi \partial_{N N} \Psi_{N N}+\partial_{A}\left(\partial_{N N} \Psi_{B N}\right)+\partial_{B}\left(\partial_{N N} \Psi_{A N}\right) \\
& -\kappa \partial_{N N} \Psi_{A B}-\xi \partial_{N N}\left(\Psi_{A A}+\Psi_{B B}\right)-\gamma^{B} \partial_{N N} \Psi_{A N}-\gamma^{A} \partial_{N N} \Psi_{B N}-\partial_{N N N} \Psi_{A B} .
\end{aligned}
$$

Thus, by (3.18) and (3.19), one has

$$
\partial_{N} W_{i j} \tau_{j}^{A} \tau_{i}^{B}=-\kappa V_{A B}+\xi\left(V_{A A}+V_{B B}\right)-\partial_{N}^{3} \Psi_{A B}=-\partial_{N} V_{A B} .
$$

- Now we address the term $\partial_{N} W N \cdot \tau^{R}$. It holds

$$
\begin{aligned}
\partial_{N} W_{i j} N_{j} \tau_{i}^{A}=\partial_{N}\left[\left(N_{m} \partial_{B}-\tau_{m}^{B} \partial_{N}\right)\left(\tau_{n}^{B} \partial_{A}-\tau_{n}^{A} \partial_{B}\right) \Psi_{m n}\right. & \\
& \left.-\epsilon_{j l n}\left(\left(N_{m} \partial_{B}+\tau_{m}^{B} \partial_{N}\right) N_{j}\right)\left(\tau_{l}^{A} \partial_{A}+\tau_{l}^{B} \partial_{B}+N_{l} \partial_{N}\right) \Psi_{m n}\right],
\end{aligned}
$$

which by (3.17) rewrites as

$$
\begin{aligned}
\partial_{N} W_{i j} N_{j} \tau_{i}^{A}=\left(N_{m} \partial_{N B}-\tau_{m}^{B} \partial_{N N}\right) & \left(\tau_{n}^{B} \partial_{A}-\tau_{n}^{A} \partial_{B}\right) \Psi_{m n} \\
& \quad-\epsilon_{j l n} N_{m}\left(\partial_{B} N_{j}\right)\left(\tau_{l}^{A} \partial_{N A}+\tau_{l}^{B} \partial_{N B}+N_{l} \partial_{N N}\right) \Psi_{m n},
\end{aligned}
$$

and by (3.17) and (3.23), as

$$
\partial_{N} W_{i j} N_{j} \tau_{i}^{A}=\left(N_{m} \partial_{B N}-\tau_{m}^{B} \partial_{N N}\right)\left(\tau_{n}^{B} \partial_{A}-\tau_{n}^{A} \partial_{B}\right) \Psi_{m n}-\epsilon_{j l n} N_{m}\left(\partial_{B} N_{j}\right) N_{l} \partial_{N N} \Psi_{m n} .
$$

The last term vanishes since $\partial_{N N} \Psi_{i N}=0$. Thus

$$
\partial_{N} W_{i j} N_{j} \tau_{i}^{A}=\left(N_{m} \partial_{B}-\tau_{m}^{B} \partial_{N}\right)\left(\tau_{n}^{B} \partial_{N A}-\tau_{n}^{A} \partial_{N B}\right) \Psi_{m n}-\gamma^{A} N_{m} \tau_{n}^{A} \partial_{N N} \Psi_{m n},
$$

and by (3.23), rewrites as

$\partial_{N} W_{i j} N_{j} \tau_{i}^{A}=\left(N_{m} \partial_{B}-\tau_{m}^{B} \partial_{N}\right)\left(\tau_{n}^{B} \partial_{A N}-\kappa^{A} \tau_{n}^{B} \partial_{A}-\xi \tau_{n}^{B} \partial_{B}-\tau_{n}^{A} \partial_{B N}+\kappa^{B} \tau_{n}^{A} \partial_{B}+\xi \tau_{n}^{A} \partial_{A}\right) \Psi_{m n}$, which by virtue (3.17) rewrites as

$$
\begin{aligned}
\partial_{N} W_{i j} N_{j} \tau_{i}^{A} & =-\tau_{m}^{B} \partial_{N}\left(\tau_{n}^{B} \partial_{A N}-\kappa^{A} \tau_{n}^{B} \partial_{A}-\xi \tau_{n}^{B} \partial_{B}-\tau_{n}^{A} \partial_{B N}+\kappa^{B} \tau_{n}^{A} \partial_{B}+\xi \tau_{n}^{A} \partial_{A}\right) \Psi_{m n} \\
& =-\tau_{m}^{B}\left(\tau_{n}^{B} \partial_{N A N}-\kappa^{A} \tau_{n}^{B} \partial_{N A}-\xi \tau_{n}^{B} \partial_{N B}-\tau_{n}^{A} \partial_{N B N}+\kappa^{B} \tau_{n}^{A} \partial_{N B}+\xi \tau_{n}^{A} \partial_{N A}\right) \Psi_{m n} .
\end{aligned}
$$

Again by (3.17) and (3.23), one writes

$\partial_{N} W_{i j} N_{j} \tau_{i}^{A}=-\tau_{m}^{B}\left(\tau_{n}^{B} \partial_{A N N}-\kappa^{A} \tau_{n}^{B} \partial_{A N}-\xi \tau_{n}^{B} \partial_{B N}-\tau_{n}^{A} \partial_{B N N}+\kappa^{B} \tau_{n}^{A} \partial_{B N}+\xi \tau_{n}^{A} \partial_{A N}\right) \Psi_{m n}$,

which, recalling (3.17), reads

$$
\begin{aligned}
\partial_{N} W_{i j} N_{j} \tau_{i}^{A} & =-\tau_{m}^{B}\left(\tau_{n}^{B} \partial_{A N N}-\tau_{n}^{A} \partial_{B N N}\right) \Psi_{m n} \\
& =-\tau_{m}^{B} \tau_{n}^{B} \partial_{A N N} \Psi_{m n}+\tau_{m}^{B} \tau_{n}^{A} \partial_{B N N} \Psi_{m n} .
\end{aligned}
$$

Yet,

$-\partial_{A}\left(\tau_{m}^{B} \tau_{n}^{B}\right)+\partial_{B}\left(\tau_{m}^{B} \tau_{n}^{A}\right)=-\gamma^{B}\left(\tau_{m}^{A} \tau_{n}^{B}+\tau_{m}^{B} \tau_{n}^{A}\right)+\xi N_{m} \tau_{n}^{B}-\kappa^{B} N_{m} \tau_{n}^{A}+\gamma^{A}\left(-\tau_{m}^{A} \tau_{n}^{A}+\tau_{m}^{B} \tau_{n}^{B}\right)$, and hence

$$
\begin{aligned}
\partial_{N} W_{i j} N_{j} \tau_{i}^{A}= & -\partial_{A}\left(\tau_{m}^{B} \tau_{n}^{B} \partial_{N N} \Psi_{m n}\right)+\partial_{B}\left(\tau_{m}^{B} \tau_{n}^{A} \partial_{N N} \Psi_{m n}\right)-\gamma^{A} N_{m} \tau_{n}^{A} \partial_{N N} \Psi_{m n} \\
& -\left(-\gamma^{B}\left(\tau_{m}^{A} \tau_{n}^{B}+\tau_{m}^{B} \tau_{n}^{A}\right)+\xi N_{m} \tau_{n}^{B}-\kappa^{B} N_{m} \tau_{n}^{A}+\gamma^{A}\left(-\tau_{m}^{A} \tau_{n}^{A}+\tau_{m}^{B} \tau_{n}^{B}\right)\right) \partial_{N N} \Psi_{m n} \\
= & -\partial_{A N N} \Psi_{B B}+\partial_{B N N} \Psi_{A B}-\gamma^{A} \partial_{N N} \Psi_{N A} \\
& +2 \gamma^{B} \partial_{N N} \Psi_{A B}-\xi \partial_{N N} \Psi_{B N}+\kappa^{B} \partial_{N N} \Psi_{A N}+\gamma^{A} \partial_{N N}\left(\Psi_{A A}-\Psi_{B B}\right) .
\end{aligned}
$$

Therefore, (3.18) yields

$$
\partial_{N} W_{i j} N_{j} \tau_{i}^{A}=\partial_{A} V_{A A}+\partial_{B} V_{A B}+2 \gamma_{B} V_{A B}+\gamma^{A}\left(V_{A A}-V_{B B}\right) .
$$


Since $\operatorname{div} V=0$ and $V N=0$, we infer from (2.16) that

$$
\partial_{N} V_{A N}+\partial_{A} V_{A A}+\gamma^{A}\left(V_{A A}-V_{B B}\right)+\partial_{B} V_{A B}+2 \gamma^{B} V_{A B}=0 .
$$

Thus

$$
\partial_{N} W_{A N}=-\partial_{N} V_{A N}
$$

Similarly one gets $\partial_{N} W_{B N}=-\partial_{N} V_{B N}$.

- Lastly it remains to consider $\partial_{N} W N \cdot N$. We have

$$
\begin{aligned}
\partial_{N} W_{i j} N_{j} N_{i}=\partial_{N}\left[\left(\tau_{m}^{B} \partial_{A}-\tau_{m}^{A} \partial_{B}\right)\right. & \left(\tau_{n}^{B} \partial_{A}-\tau_{n}^{A} \partial_{B}\right) \Psi_{m n} \\
& \left.-\epsilon_{j l n}\left(\tau_{m}^{B} \partial_{A}-\tau_{m}^{A} \partial_{B}\right) N_{j}\left(\tau_{l}^{A} \partial_{A}+\tau_{l}^{B} \partial_{B}+N_{l} \partial_{N}\right) \Psi_{m n}\right],
\end{aligned}
$$

which by (3.17) writes as

$$
\begin{aligned}
\partial_{N} W_{i j} N_{j} N_{i}=\left(\tau_{m}^{B} \partial_{N A}-\tau_{m}^{A} \partial_{N B}\right)\left(\tau_{n}^{B} \partial_{A}-\tau_{n}^{A} \partial_{B}\right) \Psi_{m n} \\
\quad-\epsilon_{j l n}\left(\tau_{m}^{B} \partial_{A}-\tau_{m}^{A} \partial_{B}\right) N_{j}\left(\tau_{l}^{A} \partial_{N A}+\tau_{l}^{B} \partial_{N B}+N_{l} \partial_{N N}\right) \Psi_{m n},
\end{aligned}
$$

and again by (3.17), recalling (3.23), rewrites as

$$
\begin{aligned}
\partial_{N} W_{i j} N_{j} N_{i}= & \left(\tau_{m}^{B} \partial_{A N}-\tau_{m}^{A} \partial_{B N}\right)\left(\tau_{n}^{B} \partial_{A}-\tau_{n}^{A} \partial_{B}\right) \Psi_{m n} \\
& -\epsilon_{j l n}\left(\kappa^{A} \tau_{m}^{B} \tau_{j}^{A}+\xi \tau_{m}^{B} \tau_{j}^{B}-\kappa^{B} \tau_{m}^{A} \tau_{j}^{B}-\xi \tau_{m}^{A} \tau_{j}^{A}\right) N_{l} \partial_{N N} \Psi_{m n} \\
= & \left(\tau_{m}^{B} \partial_{A}-\tau_{m}^{A} \partial_{B}\right)\left(\tau_{n}^{B} \partial_{N A}-\tau_{n}^{A} \partial_{N B}\right) \Psi_{m n} \\
& -\left(-\kappa^{A} \tau_{m}^{B} \tau_{n}^{B}+\xi \tau_{m}^{B} \tau_{n}^{A}-\kappa^{B} \tau_{m}^{A} \tau_{n}^{A}+\xi \tau_{m}^{A} \tau_{n}^{B}\right) \partial_{N N} \Psi_{m n} .
\end{aligned}
$$

Hence, (3.17) and (3.23) imply that

$$
\begin{aligned}
\partial_{N} W_{i j} N_{j} N_{i}= & \left(\tau_{m}^{B} \partial_{A}-\tau_{m}^{A} \partial_{B}\right)\left(\tau_{n}^{B} \partial_{A N}-\tau_{n}^{A} \partial_{A N}\right) \Psi_{m n} \\
& +\left(\kappa^{A} \tau_{m}^{B} \tau_{n}^{B}+\kappa^{B} \tau_{m}^{A} \tau_{n}^{A}-\xi \tau_{m}^{B} \tau_{n}^{A}-\xi \tau_{m}^{A} \tau_{n}^{B}\right) \partial_{N N} \Psi_{m n} .
\end{aligned}
$$

Yet, (3.17) yields

$$
\partial_{N} W_{i j} N_{j} N_{i}=\kappa^{A} \partial_{N N} \Psi_{B B}+\kappa^{B} \partial_{N N} \Psi_{A A}-2 \xi \partial_{N N} \Psi_{A B},
$$

which by (3.18) achieves the calculation, since

$$
\partial_{N} W_{i j} N_{j} N_{i}=-\kappa^{A} V_{A A}-\kappa^{B} V_{B B}-2 \xi V_{A B} .
$$

Recall now that $\operatorname{div} V=0$ and $V N=0$, to infer from (2.16) that

$$
\partial_{N} W_{N N}=-\partial_{N} V_{N N} \text {. }
$$

Thus (3.16) is satisfied.

Step 5. The proof is achieved by the classical lifting theorem in $H^{4}(\Omega)$ for the components of $\Psi$ in the local basis, such that (3.17),(3.18) and (3.19) are satisfied.

If $E$ is a symmetric matrix decomposed as (2.15), we denote by $E_{T}$ the tangential part of $E$ with components

$$
\left(E_{T}\right)_{i j}:=E_{A A} \tau_{i}^{A} \tau_{j}^{A}+E_{B B} \tau_{i}^{B} \tau_{j}^{B}+E_{A B}\left(\tau_{i}^{A} \tau_{j}^{B}+\tau_{j}^{A} \tau_{i}^{B}\right) .
$$

Lemma 3.11. Let $\mathbb{E} \in H^{3 / 2}\left(\partial \Omega, \mathbb{S}^{3}\right), \mathbb{G} \in H^{1 / 2}\left(\partial \Omega, \mathbb{S}^{3}\right)$. There exists $H \in H^{2}\left(\Omega, \mathbb{S}^{3}\right)$ such that

$$
\begin{cases}H=\mathbb{E} & \text { on } \partial \Omega, \\ \left(\partial_{N} H\right)_{T}=\mathbb{G}_{T} & \text { on } \partial \Omega, \\ \operatorname{div} H=0 & \text { on } \partial \Omega .\end{cases}
$$

Proof. By the lifting theorem in $H^{2}(\Omega)$, one constructs functions $H_{A A}, H_{A B}, H_{B B} \in H^{2}(\Omega)$ such that on $\partial \Omega$ :

$$
\begin{gathered}
H_{A A}=\mathbb{E}_{A A}, H_{A B}=\mathbb{E}_{A B}, H_{B B}=\mathbb{E}_{B B}, \\
\partial_{N} H_{A A}=\mathbb{G}_{A A}, \partial_{N} H_{A B}=\mathbb{G}_{A B}, \partial_{N} H_{B B}=\mathbb{G}_{B B} .
\end{gathered}
$$

By (2.16), the conditions $\operatorname{div} H=0$ and $H=\mathbb{E}$ on $\partial \Omega$ impose $\partial_{N} H_{N N}, \partial_{N} H_{N A}$ and $\partial_{N} H_{N B}$ on $\partial \Omega$. Then one constructs $H_{N N}, H_{N A}$ and $H_{N B}$ in $\Omega$ using again the lifting theorem in $H^{2}(\Omega)$. 
Theorem 3.12. Let $\mathbb{E} \in H^{3 / 2}\left(\partial \Omega, \mathbb{S}^{3}\right)$ with

$$
\int_{\partial \Omega} \mathbb{E} N d S(x)=0
$$

i.e., $\mathbb{E} \in \tilde{H}^{3 / 2}\left(\partial \Omega, \mathbb{S}^{3}\right)$, and $\mathbb{G} \in H^{1 / 2}\left(\partial \Omega, \mathbb{S}^{3}\right)$. There exists $E \in H^{2}\left(\Omega, \mathbb{S}^{3}\right)$ such that

$$
\begin{cases}E=\mathbb{E} & \text { on } \partial \Omega \\ \left(\partial_{N} E\right)_{T}=\mathbb{G}_{T} & \text { on } \partial \Omega \\ \operatorname{div} E=0 & \text { in } \Omega .\end{cases}
$$

In addition, such a lifting can be obtained through a linear continuous operator

$$
\mathcal{L}_{\partial \Omega}:(\mathbb{E}, \mathbb{G}) \in \tilde{H}^{3 / 2}\left(\partial \Omega, \mathbb{S}^{3}\right) \times H^{1 / 2}\left(\partial \Omega, \mathbb{S}^{3}\right) \mapsto E \in H^{2}\left(\Omega, \mathbb{S}^{3}\right) .
$$

Proof. Let $H$ be the function defined in Lemma 3.11. We must construct $K=E-H \in$ $H^{2}\left(\Omega, \mathbb{S}^{3}\right)$ satisfying

$$
\begin{cases}K=0 & \text { on } \partial \Omega \\ \left(\partial_{N} K\right)_{T}=0 & \text { on } \partial \Omega \\ \operatorname{div} K=-\operatorname{div} H & \text { in } \Omega .\end{cases}
$$

We have $\operatorname{div} H \in H_{0}^{1}\left(\Omega, \mathbb{R}^{3}\right)$ and

$$
\int_{\Omega} \operatorname{div} H d x=\int_{\partial \Omega} H N d S(x)=\int_{\partial \Omega} \mathbb{E} N d S(x)=0 .
$$

Therefore Theorem 3.10 provides the desired $K$. Finally, the linearity and the continuity of the obtained lifting is easily checked at each step of its construction.

3.3. Beltrami decomposition. The following result is again given for the sake of generality in $L^{p}(\Omega)$ with $1<p<\infty$ but should be here be considered for $p=2$.

Theorem 3.13 (Beltrami decomposition [15]). Assume that $\Omega$ is simply-connected. Let $p \in$ $(1,+\infty)$ be a real number and let $E \in L^{p}\left(\Omega, \mathbb{S}^{3}\right)$ be a symmetric tensor. Then, For any $U \in$ $W^{1 / p, p}(\partial \Omega)$, there exists a unique $u \in W^{1, p}\left(\Omega, \mathbb{R}^{3}\right)$ with $u=U$ on $\partial \Omega$ and a unique $F \in$ $L^{p}\left(\Omega, \mathbb{S}^{3}\right)$ with $\operatorname{Curl} F \in L^{p}\left(\Omega, \mathbb{R}^{3 \times 3}\right)$, inc $F \in L^{p}\left(\Omega, \mathbb{S}^{3}\right)$, $\operatorname{div} F=0$ and $F N=0$ on $\partial \Omega$ such that

$$
E=\nabla^{S} u+\operatorname{inc} F \text {. }
$$

We call $\nabla^{S} u$ the compatible part and $E^{0}:=$ inc $F$ the (solenoidal) incompatible part of the Beltrami decomposition.

3.4. Green formula. Let $V$ be a vector field defined on $\partial \Omega$ and let $\tilde{V}$ be any extension of $V$ in $\Omega$ with appropriate regularity. The surface divergence of $V$ is defined on $\partial \Omega$ by

$$
\operatorname{div}_{\mathrm{S}} V=\operatorname{div} \tilde{V}-\left(\partial_{N} \tilde{V}\right) \cdot N .
$$

Lemma 3.14 ([12]). If $V \in W^{1,1}\left(\partial \Omega, \mathbb{R}^{3}\right)$ then

$$
\int_{\partial \Omega} \operatorname{div}_{\mathrm{S}} V d S(x)=\int_{\partial \Omega} \kappa V \cdot N d S(x) .
$$

Lemma 3.15. For all $A, B \in \mathcal{C}^{2}\left(\bar{\Omega}, \mathbb{M}^{3}\right)$,

$$
\int_{\Omega} A \cdot \operatorname{Curl} B d x=\int_{\Omega} \operatorname{Curl} A \cdot B d x+\int_{\partial \Omega}(A \times N) \cdot B d S(x) .
$$

Proof. We have

$$
\begin{aligned}
\int_{\Omega} A \cdot \operatorname{Curl} B d x & =\int_{\Omega} \epsilon_{j k m} A_{i j} \partial_{k} B_{i m} d x \\
& =-\int_{\Omega} \epsilon_{j k m} \partial_{k} A_{i j} B_{i m} d x+\int_{\partial \Omega} \epsilon_{j k m} A_{i j} B_{i m} N_{k} d S(x) \\
& =\int_{\Omega}(\operatorname{Curl} A)_{i m} B_{i m} d x+\int_{\partial \Omega}(A \times N)_{i m} B_{i m} d S(x) .
\end{aligned}
$$

Denote $A^{S}=\left(A+A^{t}\right) / 2$ the symmetric part of a tensor $A$. 
Theorem 3.16. Suppose that $T \in \mathcal{C}^{2}\left(\bar{\Omega}, \mathbb{S}^{3}\right)$ and $\eta \in H^{2}\left(\Omega, \mathbb{S}^{3}\right)$. Then

$$
\begin{aligned}
\int_{\Omega} T \cdot \text { inc } \eta d x & =\int_{\Omega} \operatorname{inc} T \cdot \eta d x \\
& +\int_{\partial \Omega} \mathcal{T}_{1}(T) \cdot \eta d S(x)+\int_{\partial \Omega} \mathcal{T}_{0}(T) \cdot \partial_{N} \eta d S(x)
\end{aligned}
$$

with the trace operators defined as

$$
\begin{aligned}
& \mathcal{T}_{0}(T):=(T \times N)^{t} \times N, \\
& \mathcal{T}_{1}(T):=\left(\operatorname{Curl}(T \times N)^{t}\right)^{S}+\left(\left(\partial_{N}+\kappa\right) T \times N\right)^{t} \times N+\left(\operatorname{Curl}^{\mathrm{t}} T \times N\right)^{S} .
\end{aligned}
$$

Proof. By density we can assume that $\eta$ is smooth. Lemma 3.15 yields

$$
\int_{\Omega} T \cdot \operatorname{inc} \eta d x=\int_{\Omega} \operatorname{Curl}^{\mathrm{t}} T \cdot \operatorname{Curl} \eta d x+\int_{\partial \Omega} \operatorname{Curl} \eta \cdot(T \times N)^{t} d S(x) .
$$

From the definition of the cross product of two tensors and its trace we observe that

$$
\operatorname{div}(\operatorname{tr} A \times B)=\operatorname{Curl} A \cdot B-\operatorname{Curl} B \cdot A .
$$

As a consequence, setting $A=(T \times N)^{t}$ and $B=\eta$ in the above identity, one has

$$
\begin{array}{r}
\int_{\Omega} T \cdot \operatorname{inc} \eta d x=\int_{\Omega} \operatorname{Curl}^{\mathrm{t}} T \cdot \operatorname{Curl} \eta d x+\int_{\partial \Omega} \eta \cdot \operatorname{Curl}(T \times N)^{t} d S(x) \\
-\int_{\partial \Omega} \operatorname{div}\left(\operatorname{tr}\left((T \times N)^{t} \times \eta\right)\right) d S(x) .
\end{array}
$$

By definition of the surface divergence, this rewrites as

$$
\begin{aligned}
\int_{\Omega} T \cdot \operatorname{inc} \eta d x & =\int_{\Omega} \operatorname{Curl}^{\mathrm{t}} T \cdot \operatorname{Curl} \eta d x+\int_{\partial \Omega} \eta \cdot \operatorname{Curl}(T \times N)^{t} d S(x) \\
& -\int_{\partial \Omega}\left[\operatorname{div}_{\mathrm{S}}\left(\operatorname{tr}\left((T \times N)^{t} \times \eta\right)\right)+\partial_{N}\left(\operatorname{tr}\left((T \times N)^{t} \times \eta\right)\right) \cdot N\right] d S(x) .
\end{aligned}
$$

A short calculation shows that for two tensors $A, B$,

$$
\operatorname{tr}(A \times B) \cdot N=-(A \times N) \cdot B .
$$

Using Lemma 3.14 we obtain

$$
\begin{aligned}
\int_{\Omega} T \cdot \operatorname{inc} \eta d x=\int_{\Omega} \operatorname{Curl}^{\mathrm{t}} T \cdot \operatorname{Curl} \eta d x & +\int_{\partial \Omega} \eta \cdot \operatorname{Curl}(T \times N)^{t} d S(x) \\
& \left.\quad+\int_{\partial \Omega} \kappa(T \times N)^{t} \times N \cdot \eta d S(x)+\int_{\partial \Omega} \partial_{N}\left((T \times N)^{t} \times N\right) \cdot \eta\right) d S(x) .
\end{aligned}
$$

Rearranging yields

$$
\begin{aligned}
\int_{\Omega} T \cdot \operatorname{inc} \eta d x=\int_{\Omega} \operatorname{Curl}^{\mathrm{t}} T \cdot \operatorname{Curl} \eta d x+\int_{\partial \Omega}(T \times N)^{t} \times N \cdot \partial_{N} \eta d S(x) \\
\quad+\int_{\partial \Omega}\left(\operatorname{Curl}(T \times N)^{t}+\left(\left(\partial_{N}+\kappa\right) T \times N\right)^{t} \times N\right) \cdot \eta d S(x) .
\end{aligned}
$$

One concludes using Lemma 3.15.

Remark 3.17. By Remark 3.4, only $\left(\partial_{N} \eta\right)_{T}$ matters in the rightmost integral of (3.32).

Remark 3.18. For a symmetric tensor $A$ and vectors $u$ and $v$, one has $\left((A \times u)^{t} \times v\right)^{t}=$ $(A \times v)^{t} \times u$. Indeed, we have componentwise

$$
\left.\left((A \times u)^{t} \times v\right)_{i p}=\epsilon_{p j m} \epsilon_{i k l} A_{j k} u_{l} v_{m}=\epsilon_{i k l} \epsilon_{p j m} A_{k j} v_{m} u_{l}=\epsilon_{i k l}(A \times v)_{p k}^{t} u_{l}=(A \times v)^{t} \times u\right)_{p i} .
$$


Lemma 3.19. We have the alternative expressions

$$
\begin{array}{r}
\mathcal{T}_{1}(T)=-\sum_{R} \kappa^{R}\left(T \times \tau^{R}\right)^{t} \times \tau^{R}-\sum_{R} \xi\left(T \times \tau^{R}\right)^{t} \times \tau^{R^{*}}+\left(\left(-\partial_{N}+\kappa\right) T \times N\right)^{t} \times N \\
-2\left(\sum_{R}\left(\partial_{R} T \times N\right)^{t} \times \tau^{R}\right)^{S}, \\
\mathcal{T}_{1}(T)=\sum_{R} \kappa^{R}\left(T \times \tau^{R}\right)^{t} \times \tau^{R}+\sum_{R} \xi\left(T \times \tau^{R}\right)^{t} \times \tau^{R^{*}}-\left(\left(\partial_{N}+\kappa\right) T \times N\right)^{t} \times N \\
-2 \sum_{R}\left(\partial_{R}+\gamma^{R}\right)\left((T \times N)^{t} \times \tau^{R}\right)^{S} .
\end{array}
$$

In addition it holds

$$
\int_{\partial \Omega} \mathcal{T}_{1}(T) N d S(x)=0
$$

Proof. We have

$$
\begin{aligned}
\left(\operatorname{Curl}(T \times N)^{t}\right)_{m n}= & -\epsilon_{i k m} \epsilon_{j l n} \partial_{l}\left(N_{k} T_{i j}\right) \\
= & -\epsilon_{i k m} \epsilon_{j l n}\left(\partial_{l} N_{k} T_{i j}+N_{k} \partial_{l} T_{i j}\right) \\
= & -\epsilon_{i k m} \epsilon_{j l n}\left(\sum_{R} \tau_{l}^{R} \partial_{R} N_{k} T_{i j}+N_{k} \partial_{l} T_{i j}\right) \\
= & -\epsilon_{i k m} \epsilon_{j l n}\left(\sum_{R}\left(\kappa^{R} \tau_{l}^{R} \tau_{k}^{R}+\xi \tau_{l}^{R} \tau_{k}^{R^{*}}\right) T_{i j}+N_{k} \partial_{l} T_{i j}\right) \\
= & -\sum_{R} \kappa^{R}\left(\left(T \times \tau^{R}\right)^{t} \times \tau^{R}\right)_{n m}-\sum_{R} \xi\left(\left(T \times \tau^{R}\right)^{t} \times \tau^{R^{*}}\right)_{n m} \\
& +\left(\operatorname{Curl}^{\mathrm{t}} T \times N\right)_{n m} .
\end{aligned}
$$

Hence

$\operatorname{Curl}(T \times N)^{t}=-\sum_{R} \kappa^{R}\left(T \times \tau^{R}\right)^{t} \times \tau^{R}-\sum_{R} \xi\left(T \times \tau^{R}\right)^{t} \times \tau^{R^{*}}+\left(\operatorname{Curl}^{\mathrm{t}} T \times N\right)^{t}$.

By Lemma 3.1 and (3.35) we obtain (3.36).

Denote $E^{A}=E-E^{S}=\frac{1}{2}\left(E-E^{t}\right)$. By (3.39) and Lemma 3.1 we have

$$
\left(\operatorname{Curl}(T \times N)^{t}\right)^{A}=-\left(\operatorname{Curl}^{\mathrm{t}} T \times N\right)^{A}=\left(\sum_{R}\left(\partial_{R} T \times \tau^{R}\right)^{t} \times N\right)^{A} .
$$

Integrating against $N$ and using the Stokes formula, by which $\int_{\partial \Omega} \operatorname{Curl} F N d S(x)=0$ for any tensor $F$, yields

$$
\int_{\partial \Omega} \operatorname{Curl}^{\mathrm{t}}(T \times N)^{t} N d S(x)=\int_{\partial \Omega}\left(\sum_{R}\left(\partial_{R} T \times \tau^{R}\right)^{t} \times N\right)^{t} N d S(x) .
$$

Using (3.35) and reordering the mixed product entails

$$
\int_{\partial \Omega} \operatorname{Curl}^{\mathrm{t}}(T \times N)^{t} N d S(x)=-\int_{\partial \Omega}\left(\sum_{R}\left(\partial_{R} T \times N\right)^{t} \times N\right) \tau^{R} d S(x) .
$$

From the Stokes formula we have

$$
\int_{\partial \Omega} \mathcal{T}_{1}(T) N d S(x)=\frac{1}{2} \int_{\partial \Omega} \operatorname{Curl}^{\mathrm{t}}(T \times N)^{t} N d S(x)+\frac{1}{2} \int_{\partial \Omega}\left(\operatorname{Curl}^{\mathrm{t}} T \times N\right)^{t} N d S(x),
$$
and by Lemma 3.1

$$
\left(\operatorname{Curl}^{\mathrm{t}} T \times N\right)^{t} N=-\sum_{R}\left(\left(\partial_{R} T \times \tau^{R}\right)^{t} \times N\right)^{t} N=\sum_{R}\left(\left(\partial_{R} T \times N\right)^{t} \times N\right) \tau^{R} .
$$

Combining (3.41), (3.40) and (3.42) entails (3.38). 
Lastly, (3.37) is derived from (3.36) using

$$
\left(\partial_{R} T \times N\right)^{t} \times \tau^{R}=\partial_{R}\left((T \times N)^{t} \times \tau^{R}\right)-\left(T \times \partial_{R} N\right)^{t} \times \tau^{R}-(T \times N)^{t} \times \partial_{R} \tau^{R} .
$$

3.5. Gauge conditions. Theorem 3.16 applies to arbitrary test functions $\eta \in H^{2}\left(\Omega, \mathbb{S}^{3}\right)$, but only solenoidal fields are considered in the targeted application. The implications as to the dual characterization of the boundary term $\mathcal{T}_{1}(T)$ is discussed below. We define the gauge set

$$
\mathcal{G}:=\left\{V \odot N, V \in \mathbb{R}^{3}\right\} \subset \mathcal{C}^{\infty}\left(\partial \Omega, \mathbb{S}^{3}\right)
$$

with

$$
V_{1} \odot V_{2}:=\frac{1}{2}\left(V_{1} V_{2}^{t}+V_{2} V_{1}^{t}\right) \quad \forall V_{1}, V_{2} \in \mathbb{R}^{3},
$$

and the matrices

$$
M=\int_{\partial \Omega} N N^{t} d S(x), \quad P=\left(|\partial \Omega| \mathbb{I}_{2}+M\right)^{-1} .
$$

In all the sequel we will denote duality pairings by integrals for the sake of readability.

Lemma 3.20. Let $\mathbb{E} \in H^{-3 / 2}\left(\partial \Omega, \mathbb{S}^{3}\right)$. Then the condition

$$
\int_{\partial \Omega} \mathbb{E} \cdot F d S(x)=0 \quad \forall F \in \tilde{H}^{3 / 2}\left(\partial \Omega, \mathbb{S}^{3}\right)
$$

holds true if and only if $\mathbb{E} \in \mathcal{G}$.

Proof. Assume first that $\mathbb{E} \in \mathcal{G}$, i.e., $\mathbb{E}=V \odot N$ for some $V \in \mathbb{R}^{3}$. We have for all $F \in$ $\tilde{H}^{3 / 2}\left(\partial \Omega, \mathbb{S}^{3}\right)$

$$
\int_{\partial \Omega} \mathbb{E} \cdot F d S(x)=\int_{\partial \Omega}(V \odot N) \cdot F d S(x)=\int_{\partial \Omega}(F N) \cdot V d S(x)=0 .
$$

Assume now that $\mathbb{E} \in H^{-3 / 2}\left(\partial \Omega, \mathbb{S}^{3}\right)$ satisfies (3.43). Let $F \in H^{3 / 2}\left(\partial \Omega, \mathbb{S}^{3}\right)$ be arbitrary and define

$$
\Phi=\int_{\partial \Omega} F N d S(x), \quad \tilde{F}=F-2(P \Phi) \odot N .
$$

We have

$$
\begin{aligned}
\int_{\partial \Omega} \tilde{F} N d S(x) & =\Phi-2 \int_{\partial \Omega}((P \Phi) \odot N) N d S(x) \\
& =\Phi-\int_{\partial \Omega}\left(P \Phi N^{t} N+N N^{t} P \Phi\right) d S(x) \\
& =\Phi-(P \Phi|\partial \Omega|+M P \Phi) \\
& =\Phi-\left(|\partial \Omega| \mathbb{I}_{2}+M\right) P \Phi=0 .
\end{aligned}
$$

This implies that $\tilde{F} \in \tilde{H}^{3 / 2}\left(\partial \Omega, \mathbb{S}^{3}\right)$. Therefore

$$
\begin{aligned}
0 & =\int_{\partial \Omega} \mathbb{E} \cdot \tilde{F} d S(x) \\
& =\int_{\partial \Omega} \mathbb{E} \cdot F d S(x)-2 \int_{\partial \Omega}(P \Phi) \cdot(\mathbb{E} N) d S(x) \\
& =\int_{\partial \Omega} \mathbb{E} \cdot F d S(x)-2 P\left(\int_{\partial \Omega} F N d S(x)\right) \cdot\left(\int_{\partial \Omega} \mathbb{E} N d S(x)\right) .
\end{aligned}
$$

Set $V=P \int_{\partial \Omega} \mathbb{E} N d S(x)$. We obtain

$$
0=\int_{\partial \Omega} \mathbb{E} \cdot F d S(x)-2 V \cdot\left(\int_{\partial \Omega} F N d S(x)\right)=\int_{\partial \Omega}\left(\mathbb{E}-N V^{t}-V N^{t}\right) \cdot F d S(x) .
$$

This being true for all $F \in H^{3 / 2}\left(\partial \Omega, \mathbb{S}^{3}\right)$, we infer $\mathbb{E}=N V^{t}+V N^{t}=2 V \odot N$. 
We denote by $\left(\tilde{H}^{3 / 2}\left(\partial \Omega, \mathbb{S}^{3}\right)\right)^{\prime}$ the dual space of $\tilde{H}^{3 / 2}\left(\partial \Omega, \mathbb{S}^{3}\right)$. The restriction operator

$$
\mathcal{R}: H^{-3 / 2}\left(\partial \Omega, \mathbb{S}^{3}\right) \rightarrow\left(\tilde{H}^{3 / 2}\left(\partial \Omega, \mathbb{S}^{3}\right)\right)^{\prime}
$$

is surjective by the Hahn-Banach theorem, and Lemma 3.20 says that $\operatorname{ker} \mathcal{R}=\mathcal{G}$. Therefore, the reduced map $\tilde{\mathcal{R}}: H^{-3 / 2}\left(\partial \Omega, \mathbb{S}^{3}\right) / \mathcal{G} \rightarrow\left(\tilde{H}^{3 / 2}\left(\partial \Omega, \mathbb{S}^{3}\right)\right)^{\prime}$ is an isomorphism. This permits to identify the dual of $\tilde{H}^{3 / 2}\left(\partial \Omega, \mathbb{S}^{3}\right)$ with the quotient space $H^{-3 / 2}\left(\partial \Omega, \mathbb{S}^{3}\right) / \mathcal{G}$.

Lemma 3.21. Every $\mathbb{E} \in H^{-3 / 2}\left(\partial \Omega, \mathbb{S}^{3}\right) / \mathcal{G}$ admits a unique representative $\tilde{\mathbb{E}}$ such that

$$
\int_{\partial \Omega} \tilde{\mathbb{E}} N d S(x)=0
$$

It is given by

$$
\tilde{\mathbb{E}}=\mathbb{E}-2\left(P \int_{\partial \Omega} \mathbb{E} N d S(x)\right) \odot N
$$

Proof. Arguing as in Lemma 3.20, one obtains that the function $\tilde{\mathbb{E}}$ defined by (3.45) satisfies (3.44). For the uniqueness, one has to show that, if $\tilde{\mathbb{E}} \in \mathcal{G}$ satisfies $(3.44)$, then $\tilde{\mathbb{E}}=0$. Thus, suppose that $\tilde{\mathbb{E}}=V \odot N, V \in \mathbb{R}^{3}$. We have

$$
\int_{\partial \Omega} \tilde{\mathbb{E}} N d S(x)=\frac{1}{2} \int_{\partial \Omega}\left(V N^{t} N+N N^{t} V\right) d S(x)=\frac{1}{2} P^{-1} V,
$$

whereby (3.44) implies $V=0$ and subsequently $\tilde{\mathbb{E}}=0$.

With these elements at hand, we can now generalize Theorem 3.16 to arbitrary tensors $T \in H_{\text {inc }}\left(\Omega, \mathbb{S}^{3}\right)$. First we remark that, by density,

$$
\int_{\Omega} T \cdot \text { inc } \eta d x=\int_{\Omega} \operatorname{inc} T \cdot \eta d x
$$

for every $T \in H_{\mathrm{inc}}\left(\Omega, \mathbb{S}^{3}\right)$ and $\eta \in H^{2}\left(\Omega, \mathbb{R}^{3}\right)$ such that $\eta=\left(\partial_{N} \eta\right)_{T}=0$ on $\partial \Omega$. Then, for every $T \in H_{\text {inc }}\left(\Omega, \mathbb{S}^{3}\right)$, we define the traces $\mathcal{T}_{0}(T) \in H^{-1 / 2}\left(\partial \Omega, \mathbb{S}^{3}\right)$ and $\mathcal{T}_{1}(T) \in H^{-3 / 2}\left(\partial \Omega, \mathbb{S}^{3}\right) / \mathcal{G}$ by

$$
\begin{aligned}
& \left\langle\mathcal{T}_{0}(T), \varphi_{0}\right\rangle=\int_{\Omega} T \cdot \operatorname{inc} \eta_{0} d x-\int_{\Omega} \operatorname{inc} T \cdot \eta_{0} d x, \quad \forall \varphi_{0} \in H^{1 / 2}\left(\partial \Omega, \mathbb{S}^{3}\right), \\
& \left\langle\mathcal{T}_{1}(T), \varphi_{1}\right\rangle=\int_{\Omega} T \cdot \operatorname{inc} \eta_{1} d x-\int_{\Omega} \operatorname{inc} T \cdot \eta_{1} d x, \quad \forall \varphi_{1} \in \tilde{H}^{3 / 2}\left(\partial \Omega, \mathbb{S}^{3}\right),
\end{aligned}
$$

with $\eta_{0}=\mathcal{L}_{\partial \Omega}\left(0, \varphi_{0}\right)$ and $\eta_{1}=\mathcal{L}_{\partial \Omega}\left(\varphi_{1}, 0\right)$ (recall that $\mathcal{L}_{\partial \Omega}$ is the lifting operator defined in Theorem 3.12). These definitions are independent of the choice of the liftings by virtue of (3.46). In addition, by Lemma 3.21, the function $\mathcal{T}_{1}(T)$ satisfying (3.38) is unambiguously defined in this way. By linearity of $\mathcal{L}_{\partial \Omega}$, this extends formula (3.32) to any functions $T \in H_{\text {inc }}\left(\Omega, \mathbb{S}^{3}\right)$ and $\eta \in \mathcal{H}(\Omega)$.

Because of the aforementioned gauge properties, Lemmas 3.20 and 3.21 are also crucial in order to derive strong formulations. This issue is examined in the next section.

\section{A BOUNDARY VALUE PROBLEM FOR THE INCOMPATIBILITY}

In this section we assume that $\Omega$ is simply-connected.

4.1. Governing equations. Let $\alpha \in L^{\infty}(\Omega)$ with $\inf _{\Omega} \alpha>0, \mathbb{G} \in L^{2}\left(\Omega, \mathbb{S}^{3}\right)$ with $\operatorname{div} \mathbb{G}=0$ in the sense of distributions. Consider the strictly convex minimization problem

$$
\min _{E \in \mathcal{H}_{0}(\Omega)} \int_{\Omega}\left(\frac{\alpha}{2}|\operatorname{inc} E|^{2}-\mathbb{G} \cdot E\right) d x
$$

whose Euler-Lagrange equation is

$$
\int_{\Omega} \alpha \text { inc } E \cdot \operatorname{inc} F d x=\int_{\Omega} \mathbb{G} \cdot F d x \quad \forall F \in \mathcal{H}_{0}(\Omega) .
$$

By Theorem 3.9 and the Lax-Milgram theorem, (4.2) admits a unique solution $E \in \mathcal{H}_{0}(\Omega)$.

Remark that by Theorem 3.12, (4.2) also admits a unique solution in $\mathcal{H}_{\mathbb{E}, \mathbb{F}}(\Omega)$ and even in $\mathcal{H}_{\mathbb{E}, \mathbb{F} ; \Gamma_{0}}(\Omega)$ (with the test function $F \in \mathcal{H}_{\Gamma_{0}}(\Omega)$ ). In fact it suffices to consider Theorem 3.9 
and the Lax-Milgram theorem with the unknown $E-\mathcal{L}_{\partial \Omega}(\mathbb{E}, \mathbb{F})$ which satisfies homogeneous boundary conditions. Note that by (3.7), the tangential components of $\mathbb{F}$ are permutations of the components of $\left(\partial_{N} \mathbb{F}\right)_{T}$ of Theorem 3.12. It should also be remarked that by the Green formula (3.32) and arguing as in Section 4.2, the solution $E \in \mathcal{H}_{\mathbb{E}, \mathbb{F} ; \Gamma_{0}}(\Omega)$ satisfies the Neumann conditions $\mathcal{T}_{0}(\alpha$ inc $E)=\mathcal{T}_{1}(\alpha$ inc $E)=0$ on $\partial \Omega \backslash \Gamma_{0}$.

If $F \in \mathcal{D}\left(\Omega, \mathbb{S}^{3}\right)$ is not solenoidal, the Beltrami decomposition gives $F=F^{0}+\nabla^{S} w$ with $\operatorname{div} F^{0}=0$ and $w=0$ on $\partial \Omega$, and subsequently

$$
\begin{aligned}
\int_{\Omega} \alpha \text { inc } E \cdot \operatorname{inc} F d x & =\int_{\Omega} \alpha \text { inc } E \cdot \text { inc } F^{0} d x=\int_{\Omega} \mathbb{G} \cdot F^{0} d x \\
& =\int_{\Omega} \mathbb{G} \cdot F d x-\int_{\Omega} \mathbb{G} \cdot \nabla^{S} w d x=\int_{\Omega} \mathbb{G} \cdot F d x
\end{aligned}
$$

since $\operatorname{div} \mathbb{G}=0$. Thus, in view of (3.32) with $T=\alpha$ inc $E$, the strong form associated with (4.3) is:

$$
\begin{cases}\operatorname{inc}(\alpha \operatorname{inc} E)=\mathbb{G} & \text { in } \Omega, \\ \operatorname{div} E=0 & \text { in } \Omega, \\ E=0 & \text { on } \partial \Omega, \\ \left(\partial_{N} E \times N\right)^{t} \times N=0 & \text { on } \partial \Omega .\end{cases}
$$

Let us first focus on the specific case where $\alpha$ is constant. We have

Lemma 4.1. For all $E$ symmetric and solenoidal, it holds inc (inc $E)=\Delta \Delta E$.

Proof. Componentwise, one computes

$$
\begin{aligned}
(\text { inc }(\text { inc } E))_{i j} & =\varepsilon_{i k l} \varepsilon_{j m n} \varepsilon_{l p q} \varepsilon_{n r s} \partial_{k} \partial_{m} \partial_{p} \partial_{r} E_{q s} \\
& =\left(\delta_{i p} \delta_{k q}-\delta_{i q} \delta_{k p}\right)\left(\delta_{j r} \delta_{m s}-\delta_{j s} \delta_{m r}\right) \partial_{k} \partial_{m} \partial_{p} \partial_{r} E_{q s} \\
& =\partial_{i} \partial_{j} \partial_{q} \partial_{s} E_{q s}-\partial_{k}^{2} \partial_{j} \partial_{s} E_{i s}-\partial_{k}^{2} \partial_{r} \partial_{j} E_{i j}+\partial_{p}^{2} \partial_{r}^{2} E_{i j}=\Delta \Delta E_{i j},
\end{aligned}
$$

which gives the expected result.

By (3.6) and (3.7), the expression $\left(\partial_{N} E \times N\right)^{t} \times N$ is a mere linear recombination of $\partial_{N} E \times N$, whereby these two expressions are equivalent. Therefore, it is not difficult to see [21] that (4.4) for $\alpha$ constant is equivalent to

$$
\left\{\begin{array}{cccc}
\Delta(\alpha \Delta E) & =\mathbb{G} & \text { in } & \Omega, \\
E & =0 & \text { on } & \partial \Omega, \\
\operatorname{div} E & =0 & \text { on } & \partial \Omega, \\
\partial_{N} E \times N & =0 & \text { on } & \partial \Omega, \\
\partial_{N} \operatorname{div} E & =0 & \text { on } & \partial \Omega .
\end{array}\right.
$$

Moreover one has the following result [21].

Theorem 4.2. The system (4.5) admits a unique strong solution $E \in H^{4}\left(\Omega, \mathbb{S}^{3}\right)$, which is also solution of (4.2) and (4.4).

We infer the following property, which enables to reconstruct a solenoidal tensor field from its incompatibility.

Lemma 4.3. Let $T \in L^{2}\left(\Omega, \mathbb{S}^{3}\right)$ with $\operatorname{div} T=0$ in the sense of distributions. There exists $S \in H^{2}\left(\Omega, \mathbb{S}^{3}\right)$ such that $\operatorname{div} S=0$ and inc $S=T$.

Proof. It suffices to set $S=$ inc $E$ with $E$ solution of (4.4) with $\alpha=1$ and $\mathbb{G}=T$. Theorem 4.2 shows that $E \in H^{4}\left(\Omega, \mathbb{S}^{3}\right)$, hence $S \in H^{2}\left(\Omega, \mathbb{S}^{3}\right)$.

4.2. Transmission conditions. Let $\omega \subset \subset \Omega$ with smooth boundary $\partial \omega$ and outward unit normal $N$. Suppose that

$$
\alpha= \begin{cases}\alpha_{0} & \text { in } \Omega \backslash \omega \\ \alpha_{1} & \text { in } \omega\end{cases}
$$

with $\alpha_{0}, \alpha_{1}$ two positive constants. 
Theorem 4.4. Assume $E$ is solution of (4.2) and denote $T=$ inc E. Denote $\llbracket A \rrbracket$ the jump of the quantity $A$ across $\partial \omega$, with the trace counted positively on the interior side of $\omega$. Then

$$
\begin{array}{cc}
\text { inc }(\alpha T)=\mathbb{G} & \text { in } \Omega \backslash \partial \omega, \\
\llbracket \mathcal{T}_{1}(\alpha T) \rrbracket=0 & \text { on } \partial \omega, \\
\llbracket \mathcal{T}_{0}(\alpha T) \rrbracket=0 & \text { on } \partial \omega, \\
\llbracket T N \rrbracket=0 & \text { on } \partial \omega .
\end{array}
$$

Conversely, if $T \in H_{\mathrm{inc}}\left(\Omega \backslash \partial \omega, \mathbb{S}^{3}\right)$ satisfies

$$
\operatorname{div} T=0 \text { in } \Omega \backslash \partial \omega,
$$

together with (4.6)-(4.9), then

$$
\operatorname{div} T=0 \text { and inc }(\alpha T)=\mathbb{G} \text { in } \Omega
$$

in the sense of distributions. Moreover, there exists $E \in H^{2}\left(\Omega, \mathbb{S}^{3}\right)$ with $\operatorname{div} E=0$ such that $T=\operatorname{inc} E$.

Proof. Using (3.32) (in its generalized version, see the discussion in Section 3.4) we have for all $F \in \mathcal{H}_{0}(\Omega)$

$$
\begin{aligned}
\int_{\Omega} \mathbb{G} \cdot F d x & =\int_{\omega} \alpha_{1} T \cdot \operatorname{inc} F d x+\int_{\Omega \backslash \bar{\omega}} \alpha_{0} T \cdot \operatorname{inc} F d x \\
& =\int_{\omega} \operatorname{inc}\left(\alpha_{1} T\right) \cdot F d x+\int_{\Omega \backslash \bar{\omega}} \operatorname{inc}\left(\alpha_{0} T\right) \cdot F d x \\
& +\int_{\partial \omega} \llbracket \mathcal{T}_{1}(\alpha T) \rrbracket \cdot F d S(x)+\int_{\partial \omega} \llbracket \mathcal{T}_{0}(\alpha T) \rrbracket \cdot \partial_{N} F d S(x) .
\end{aligned}
$$

Choosing $F$ with compact support in $\omega$ then in $\Omega \backslash \omega$ yields (4.6), as in (4.4). By Theorem 3.12 combined with Lemmas 3.19, 3.20 and 3.21, we infer the two transmission conditions (4.7) and (4.8). In addition, one has $\operatorname{div} T=0$ which reads in the weak form

$$
\int_{\Omega} T \cdot \nabla F d x=0 \quad \forall F \in \mathcal{D}\left(\Omega, \mathbb{R}^{3}\right) .
$$

Integrating by parts yields (4.9).

The converse relies on the standard Green formula, Theorem 3.16 and Lemma 4.3.

\section{Physical interpretation}

The aim of this section is to describe two physically motivated problems where our model 4thorder boundary value problem with the inc operator is considered. In the first example special emphasis is given to the two Dirichlet boundary conditions, whereas in the second example, the main concern is the first Neumann boundary condition. In both case, providing two Dirichlet boundary conditions on (arbitrarily small, but non flat) $\Gamma_{0}$, is mandatory to ensure uniqueness of the solution.

The displacement in linear elasticity in the presence of dislocations. Let us assume that the distribution of dislocations is known and given by the smooth 2nd-rank tensor $\Lambda$ satisfying a local conservation law expressed in the form $\operatorname{div} \Lambda^{t}=0$, and meaning that the dislocation lines are closed or end at the boundary $[14,23]$. Let $\Gamma_{0}$ be a subset of $\partial \Omega$ which is not everywhere flat and has nonzero $\mathcal{H}^{2}$-measure. Let $\mathbb{F} \in H^{1 / 2}\left(\partial \Omega ; \mathbb{S}^{3}\right)$ such that $\mathbb{F} N=0$. By Lemma 3.1, one rewrites (1.7) with the second Dirichlet boundary condition restricted to $\Gamma_{0}$ and nonhomogeneous as

$$
\left\{\begin{array}{ccc}
\text { inc inc } E & = & \text { Curl } \kappa \text { in } \Omega, \\
E & = & 0 \text { on } \partial \Omega, \\
\operatorname{Curl}^{\mathrm{t} E} E N & = & -\mathbb{F} \text { on } \Gamma_{0} .
\end{array}\right.
$$


It is understood that natural (homogeneous Neumann) boundary conditions complement this system. It is first observed from (1.4) that Curl $\kappa$ is symmetric as soon as $\Lambda^{t}$ is divergence free, since its skewsymmetric part vanishes, as seen by the following computation:

$$
\begin{aligned}
\epsilon_{m n p} \epsilon_{n k l} \partial_{k}(\kappa)_{m l} & =\epsilon_{m n p} \epsilon_{n k l} \partial_{k}(\Lambda)_{m l}-\frac{1}{2} \epsilon_{m n p} \epsilon_{m n k} \partial_{k}(\Lambda)_{q q} \\
& =\partial_{p}(\Lambda)_{l l}-\partial_{m}(\Lambda)_{m p}-\frac{1}{2} \epsilon_{m n p} \epsilon_{m n k} \partial_{k}(\Lambda)_{q q}=0
\end{aligned}
$$

where we have used the identity $\epsilon_{m n p} \epsilon_{m n k}=2 \delta_{p k}$. Moreover, the second boundary condition is on the Frank tensor, the physical meaning of which is alluded to in the introduction.

Recall (3.2) and define

$$
\mathcal{H}_{\mathbb{F} ; \Gamma_{0}}(\Omega):=\left\{E \in \mathcal{H}_{0, \mathbb{F} ; \Gamma_{0}}(\Omega): E=0 \text { on } \partial \Omega\right\} .
$$

Therefore, by our existence result for the nonhomogeneous problem, the field $E$ is found as the solution of

$$
\min _{E \in \mathcal{H}_{\mathbb{F} ; \Gamma_{0}}(\Omega)} \int_{\Omega}\left(\frac{1}{2} \mid \text { inc }\left.E\right|^{2}-\operatorname{Curl} \kappa \cdot E\right) d x .
$$

Let us denote $\epsilon^{0}:=$ inc $E$. Now, by (1.6), one infers that the displacement $u$ is solution of

$$
\left\{\begin{array}{ccc}
-\operatorname{div}\left(A \nabla^{S} u\right) & =\lambda \nabla \operatorname{tr} \epsilon^{0} \quad \text { in } \Omega, \\
\left(A \nabla^{S} u\right) N & =g-\lambda \operatorname{tr} \epsilon^{0} N \text { on } \partial \Omega .
\end{array}\right.
$$

In this equation, one identifies $\lambda \nabla \operatorname{tr} \epsilon^{0}$ as a dislocation-induced conservative force in the body, and $-\lambda \operatorname{tr} \epsilon^{0} N$ as a dislocation-induced traction at the boundary.

Remark that by Lemma 3.32 one has $\mathcal{T}_{0}\left(\epsilon^{0}\right)=0$ on $\partial \Omega \backslash \Gamma_{0}$, that is, the tangential components of $\epsilon^{0}$ vanish. Obviously one can take $\Gamma_{0}=\partial \Omega$ to recover the full pure Dirichlet problem. It should also be noted that by Remark 3.5, taking $\mathbb{F}=0$ in the second Dirichlet condition implies that (inc $E) N=\epsilon^{0} N=0$ on $\Gamma_{0}$.

To summarize, in this section we have given a meaning to equation

$$
\left\{\begin{array}{ccc}
-\operatorname{div}\left(A \nabla^{S} u\right) & =f \text { in } \Omega \\
\left(A \nabla^{S} u\right) N & =g \text { on } \partial \Omega
\end{array}\right.
$$

where $u$ is the displacement field and $f$ a conservative force (as the gravity), in the case where $-\operatorname{div} \sigma=0$ (global equilibrium) and in the presence of dislocations, i.e., $\nabla^{S} u=A^{-1} \sigma-\epsilon^{0}$ with inc $\epsilon^{0}$ related to the density of dislocations. Thus, we have started with the strain as variable, as in the intrinsic models of elasticity, and then introduced the displacement as the solutions of PDEs which describe the static problem of an elastic body with dislocations.

Elements of a thermodynamic model for crystal growth. Assume that the elastic body with dislocations is embedded in an environment whose temperature field $T$ is known. Assume that the dislocation density and hence the contortion tensor satisfy a constitutive law of the type

$$
\kappa(T)=\mathbb{K}_{\mathrm{eq}} \exp \left(\frac{H}{k_{b} T_{0}}\left(\frac{T_{0}}{T}-1\right)+S\left(1-\frac{T}{T_{0}}\right)\left(\frac{T_{0}}{T}-1\right)\right),
$$

where $k_{b}$ is the Boltzman constant, $\mathbb{K}_{\mathrm{eq}}$ is the equilibrium concentration at the reference temperature $T_{0}$, and with $H$ and $S$ the effective formation enthalpy and entropy, respectively. A law such as (5.8) has been used successfully for the numerical simulation of points defects in single crystals, as reported in [22]. Referring to the brief discussion in the introduction and to Theorem 3.16, we would like to consider the mixed problem

$$
\left\{\begin{array}{ccc}
\operatorname{inc}\left(\mathbb{M} \text { inc } \epsilon^{0}\right) & = & \mathbb{G} \text { in } \Omega, \\
\epsilon^{0} & = & 0 \text { on } \partial \Omega, \\
\operatorname{Curl}^{t} \epsilon^{0} \times N & = & -\mathbb{F} \text { on } \Gamma_{0}, \\
\mathcal{T}_{0}\left(\text { inc } \epsilon^{0}\right) & = & \mathbb{T} \text { on } \partial \Omega \backslash \Gamma_{0},
\end{array}\right.
$$

whose solution exists and is unique in $\mathcal{H}_{\mathbb{F} ; \Gamma_{0}}(\Omega)$ as defined in (5.4). Our aim is to physically interpret the boundary conditions of (5.9) and in particular the Neumann condition. Before all, the first Dirichlet boundary condition means by Beltrami decomposition that

$$
\sigma N=(A \epsilon) N=\left(A \nabla^{S} u\right) N=g,
$$


on $\partial \Omega$, i.e., pure traction is exerted, with $u$ interpreted as the displacement field. As for the second, taking $\mathbb{F}=0$ implies that $\left(\right.$ inc $\left.\epsilon^{0}\right) N=(\operatorname{Curl} \kappa) N=0$ on $\Gamma_{0}$. By $(5.8)$ this rewrites as $\kappa^{\prime}\left(T_{0}\right)(\nabla T \times N)=0$ with $T_{0}$ the temperature at $\Gamma_{0}$, which is a condition satisfied if and only if $T=T_{0}$ is a constant on $\Gamma_{0}$, that is, the temperature gradient is purely normal on $\Gamma_{0}$. As for the first Neumann boundary conditions, one has $\mathcal{T}_{0}(\operatorname{Curl} \kappa)=(\operatorname{Curl} \kappa \times N)^{t} \times N=\mathbb{T}$. By the symmetry property of $\kappa$ (cf. (5.2)), by Lemma 3.1 and by (3.35), one has

$$
(\operatorname{Curl} \kappa \times N)^{t}=\left(\operatorname{Curl}^{\mathrm{t}} \kappa \times N\right)^{t}=-\left(\partial_{N} \kappa \times N\right)^{t} \times N-\sum_{R}\left(\partial_{R} \kappa \times N\right)^{t} \times \tau^{R} .
$$

By (5.8), this yields

$$
(\operatorname{Curl} \kappa \times N)^{t}=-\partial_{N} T\left(\kappa^{\prime}(T) \times N\right)^{t} \times N-\left(\kappa^{\prime}(T) \times N\right)^{t} \times \nabla_{S} T,
$$

where $\nabla_{S} T=\sum_{R} \tau^{R} \partial_{R} T$ means the surface gradient. Thus

$$
(\operatorname{Curl} \kappa \times N)^{t} \times N=\partial_{N} T\left(\kappa^{\prime}(T) \times N\right)^{t}+\left(\left(\kappa^{\prime}(T) \times N\right)^{t} \times N\right) \times \nabla_{S} T .
$$

We have assumed that the temperature field is known and hence $\mathbb{T}$ must be given by the RHS of (5.10), which involves the normal and tangential gradients of $T$. Note that the tangential gradient may not be zero, if one thinks of a physical experiment such as Czochralski growth of single crystals $[16,22]$. Moreover, in this case one can take as $\Gamma_{0}$ the solidification interface, where on the one hand the temperature is constant (and equal to the solidification temperature $T_{0}$ ), and which on the other hand is nowhere flat (by superficial tension properties).

\section{REFERENCES}

[1] S. Agmon, A. Douglis, and L. Nirenberg. Estimates near the boundary for solutions of elliptic partial differential equations satisfying general boundary conditions. II. Commun. Pure Appl. Math., 17:35-92, 1964.

[2] S. Amstutz and A. A. Novotny. Topological asymptotic analysis of the Kirchhoff plate bending problem. ESAIM, Control Optim. Calc. Var., 17(3):705-721, 2011.

[3] S. Amstutz, A. A. Novotny, and N. Van Goethem. Topological sensitivity analysis for elliptic differential operators of order 2m. J. Differ. Equations, 256(4):1735-1770, 2014.

[4] V. Berdichevsky. Continuum theory of dislocations revisited. Cont. Mech. Therm., 18(9):195-222, 2006.

[5] Ch. Bernardi, M. Dauge, and Y. Maday. The lifting of polynomial traces revisited. Math. Comput., 79(269):47-69, 2010.

[6] Ph. G. Ciarlet and C. Mardare. Intrinsic formulation of the displacement-traction problem in linearized elasticity. Math. Models Methods Appl. Sci., 24(6):1197-1216, 2014.

[7] Philippe G. Ciarlet. An introduction to differential geometry with applications to elasticity. Springer, Dordrecht, 2005. Reprinted from J. Elasticity 78/79 (2005), no. 1-3 [MR2196098].

[8] M. C. Delfour and J.-P. Zolésio. Shapes and geometries, volume 4 of Advances in Design and Control. Society for Industrial and Applied Mathematics (SIAM), Philadelphia, PA, 2001. Analysis, differential calculus, and optimization.

[9] B. A. Dubrovin, A. T. Fomenko, and S. P. Novikov. Modern geometry - methods and applications, Part 1 (2nd edn). Cambridge studies in advanced mathematics. Springer-Verlag, New-York, 1992.

[10] G. P. Galdi. An introduction to the mathematical theory of the Navier-Stokes equations. Steady-state problems. 2nd ed. New York, NY: Springer., 2011.

[11] V. Girault and P. A. Raviart. Finite Element Methods for Navier-Stokes Equations: Theory and Algorithms. Springer Series in Computational Mathematics. Springer Berlin Heidelberg, 2011.

[12] A. Henrot and M. Pierre. Variation et optimisation de formes, volume 48 of Mathématiques 8 Applications (Berlin) [Mathematics \&s Applications]. Springer, Berlin.

[13] H. Kozono and T. Yanagisawa. $L^{r}$-variational inequality for vector fields and the Helmholtz-Weyl decomposition in bounded domains. Indiana Univ. Math. J., 58(4):1853-1920, 2009.

[14] E. Kröner. Continuum theory of defects. In R. Balian, editor, Physiques des défauts, Les Houches session XXXV (Course 3). North-Holland, Amsterdam, 1980.

[15] G. Maggiani, R. Scala, and N. Van Goethem. A compatible-incompatible decomposition of symmetric tensors in $L^{p}$ with application to elasticity. Math. Meth. Appl. Sci, 2015.

[16] G. Müller and J. Friedrich. Challenges in modeling of bulk crystal growth. J. Cryst. Growth, 266(1-3):1-19, 2004.

[17] J. F. Nye. Some geometrical relations in dislocated crystals. Acta Metall, 1:153-162, 1953.

[18] J. A. Schouten. Ricci-Calculus (2nd edn). Springer Verlag, Berlin, 1954.

[19] R. Temam. Navier-Stokes Equations: Theory 8 Numerical Analysis. Studies in Mathematics and Its Applications. North-Holland, 1984.

[20] N. Van Goethem. The non-Riemannian dislocated crystal: a tribute to Ekkehart Kröner's (1919-2000). J. Geom. Mech., 2(3), 2010. 
[21] N. Van Goethem. Cauchy elasticity with dislocations in the small strain assumption. Appl. Math. Lett., 2015.

[22] N. Van Goethem, A. de Potter, N. Van den Bogaert, and F. Dupret. Dynamic prediction of point defects in Czochralski silicon growth. An attempt to reconcile experimental defect diffusion coefficients with the $V / G$ criterion. J.Phys.Chem.Solids, 69:320-324, 2008.

[23] N. Van Goethem and F. Dupret. A distributional approach to $2 D$ Volterra dislocations at the continuum scale. Europ. Jnl. Appl. Math., 23(3):417-439, 2012

[24] W. von Wahl. Estimating $\nabla u$ by div $u$ and curl $u$. Math. Methods Appl. Sci., 15(2):123-143, 1992.

Laboratoire de Mathématiques d’Avignon, Faculté des Sciences, 33 rue Louis Pasteur, 84000 AviGNON, FRANCE

E-mail address: samuel.amstutz@univ-avignon.fr

Universidade de Lisboa, Faculdade de Ciências, Departamento de Matemática, CMAF+CiO, Alameda

da Universidade, C6, 1749-016 Lisboa, Portugal

E-mail address: vangoeth@fc.ul.pt 